\title{
Screening of antibacterial compounds with novel structure from the FDA approved drugs using machine learning methods
}

\author{
Wen-Xing $\mathrm{Li}^{1,2}$, Xin Tong ${ }^{3}$, Peng-Peng Yang ${ }^{3}$, Yang Zheng ${ }^{3}$, Ji-Hao Liang ${ }^{3}$, Gong-Hua $\mathrm{Li}^{4}$, Dahai \\ Liu $^{5}$, Dao-Gang Guan ${ }^{1,2}$, Shao-Xing Dai ${ }^{3}$ \\ ${ }^{1}$ Department of Biochemistry and Molecular Biology, School of Basic Medical Sciences, Southern Medical \\ University, Guangzhou 510515, Guangdong, China \\ ${ }^{2}$ Guangdong Provincial Key Laboratory of Single Cell Technology and Application, Southern Medical University, \\ Guangzhou 510515, Guangdong, China \\ ${ }^{3}$ State Key Laboratory of Primate Biomedical Research, Institute of Primate Translational Medicine, Kunming \\ University of Science and Technology, Kunming 650500, Yunnan, China \\ ${ }^{4}$ State Key Laboratory of Genetic Resources and Evolution, Kunming Institute of Zoology, Chinese Academy of \\ Sciences, Kunming 650223, Yunnan, China \\ ${ }^{5}$ School of Medicine, Foshan University, Foshan 528000, Guangdong, China
}

Correspondence to: Dahai Liu, Dao-Gang Guan, Shao-Xing Dai; email: dliu@fosu.edu.cn; guandg0929@hotmail.com, https://orcid.org/0000-0003-1414-0189; daisx@|pbr.cn

Keywords: antibacterial compound, drug repositioning, machine learning, structural similarity, virtual screening

Received: November 27, $2021 \quad$ Accepted: January 28, $2022 \quad$ Published: February 12, 2022

Copyright: (C) $2022 \mathrm{Li}$ et al. This is an open access article distributed under the terms of the Creative Commons Attribution License (CC BY 3.0), which permits unrestricted use, distribution, and reproduction in any medium, provided the original author and source are credited.

\section{ABSTRACT}

Bacterial infection is one of the most important factors affecting the human life span. Elderly people are more harmed by bacterial infections due to their deficits in immunity. Because of the lack of new antibiotics in recent years, bacterial resistance has increasingly become a serious problem globally. In this study, an antibacterial compound predictor was constructed using the support vector machines and random forest methods and the data of the active and inactive antibacterial compounds from the ChEMBL database. The results showed that both models have excellent prediction performance (mean accuracy $>0.9$ and mean AUC $>0.9$ for the two models). We used the predictor to screen potential antibacterial compounds from FDA-approved drugs in the DrugBank database. The screening results showed that 1087 small-molecule drugs have potential antibacterial activity and 154 of them are FDA-approved antibacterial drugs, which accounts for $76.2 \%$ of the approved antibacterial drugs collected in this study. Through molecular fingerprint similarity analysis and common substructure analysis, we screened 8 predicted antibacterial small-molecule compounds with novel structures compared with known antibacterial drugs, and 5 of them are widely used in the treatment of various tumors. This study provides a new insight for predicting antibacterial compounds by using approved drugs, the predicted compounds might be used to treat bacterial infections and extend lifespan.

\section{INTRODUCTION}

Due to deficits in innate immunity and adaptive immunity in older adults, they are more susceptible to viral and bacterial infection and experience higher incidence and severity of infectious diseases [1]. Bacterial infections may also cause common neurodegenerative disorders [2], such as Alzheimer's disease [3]. Antibiotics are a fast and effective way to deal with bacterial infections. However, with the widespread use of antibiotics, bacteria are also constantly evolving and a large number of pathogens have emerged that can resist these drugs [4]. As the research and development of novel antibiotics by 
pharmaceutical companies has drastically decreased in recent years, bacterial resistance has increasingly become a serious problem [5]. Therefore, the development of novel and highly efficient antibiotics is an urgent issue. High-throughput screening has been the dominant approach of antimicrobial drug development in the industry in the past few decades $[6,7]$. However, due to the long development time, huge cost, and low efficiency of this method [8], computer-aided drug design techniques have become a promising method in the discovery of novel antibacterial drugs [9].

Previous studies have developed multiple computational methods for efficiently assessing and screening compounds for their antimicrobial activity, such as multitarget and multi-objective approaches [10, 11]. Quantitative structure-activity relationships (QSAR) modeling is one of the most frequently employed in silico techniques for antibacterial activity prediction, improved models such as mt-QSAR and QSAR-Co can integrate multi-dimensionally heterogeneous chemical and biological data which greatly improved the reliability of such modeling [12]. The current view holds that drugs are inherently poly-pharmacological because they can act on multiple targets or disease pathways, and thus the drug discovery process should attempt to optimize more properties simultaneously [13]. Based on this theory, the multi-task model constructed by comprehensively considering the antibacterial activity of the compound and ADMET (absorption, distribution, metabolism, excretion, toxicity) characteristics can accurately screen antimycobacterial drugs [14]. Molecular fingerprints are a way of encoding molecular structure that digitizes the structural information of a compound, which are widely used in drug discovery and virtual screening [15]. Compared to other virtual screening methods, molecular fingerprints require minimal setup and configuration, are easy to calculate, and are less CPU-intensive and memory-intensive, which become the preferred tool for characterizing small molecules [16].

In recent years, machine learning methods have shown tremendous potential in the process of drug discovery and development [17]. Multiple machine learning-based methods effectively improved the accuracy of drugtarget interaction prediction [18]. Especially in the early phases of drug discovery, the use of machine learning methods significantly reduces time and effort in drug discovery and development [19]. In other areas of drug discovery, deep learning is a promising method for the prediction of molecular properties and the de novo generation of suggestions for new molecules [20]. Compared with traditional methods, machine learning approaches have the advantages of high precision, low cost, and strong operability. These technologies may have fundamentally changed the process of identifying new molecules and/or repurposing old drugs [21]. Multiple machine learning methods are widely used in ligand-based and receptor-based antibacterial drug discovery [9, 22-27]. By using chemoinformatics methods to extract the molecular characteristics of short peptides, studies have shown that the support vector machine (SVM) model can accurately predict the antibacterial activity of short peptides $[22,23]$ and the genetic characteristics of antibiotic resistance in specific pathogens [28]. The combination using random forest (RF) and genome-based analysis approaches promoted phenotypic antibacterial drug discovery [24] and revealed potential antibiotic resistance genes [25]. In recent years, emerging deep neural network methods have facilitated the discovery of antibacterial molecules with unique structures from massive data [26]. Furthermore, due to the limitations of a single method, the combination using multiple machine learning methods showed excellent performance in antibacterial compounds discovery [26, 27] and predicting the bacterial genetic mutations on drug resistance [29].

Although the popularization of machine learning methods has greatly shortened the discovery of antibacterial lead compounds, there are still required long-term studies from the identified lead compounds to clinical applications, especially experiment on drug safety [30]. Therefore, a new use for old drugs may be a way to resolve current antibiotic resistance [31]. The current Food and Drug Administration (FDA) approved antibiotics can be divided into multiple categories according to the core scaffolds, and a variety of semisynthetic antibiotics are based on these scaffolds [32]. Due to the increased bacterial resistance to specific scaffold structures, it is a promising way to develop antibiotics with novel structures. In this study, we combined using multiple machine learning methods and molecular fingerprints of compounds to build the antibacterial compound predictor and then identified structure novel small-molecule antibacterial compounds from the FDA-approved drugs.

\section{RESULTS}

\section{Initial screening of machine learning methods}

To choose the appropriate machine learning methods to construct the anti-bacterial compound prediction model, we evaluate the predictive performance of different machine learning methods including k-nearest neighbor $(\mathrm{KNN})$, logistic regression (LR), linear support vector classifier (LSVC), random forest (RF), gradient boosting regression tree (GBRT), support vector machine (SVM), and multi-layer perception (MLP). In the initial screening process, each machine learning 
method used benchmark datasets constructed from different molecular fingerprints for training and prediction with default parameters. The benchmark dataset was split into the training set (accounting for $80 \%$ ) and the validation set (accounting for $20 \%$ ), and then performed a 5-fold cross-validation test. The results suggested that the benchmark dataset based on FP2 molecular fingerprints, along with the SVM, RF, and MLP methods showed excellent prediction accuracy among all machine learning methods and molecular fingerprints combinations, whereas the accuracy fluctuates greatly among different machine learning methods in the benchmark dataset based on vector features (Figure 1). Therefore, the benchmark dataset based on FP2 molecular fingerprints, and the RF, SVM, and MLP methods were selected in the subsequent analysis.

\section{The development process of antibacterial compound predictor}

The development process of the antibacterial compound predictor is shown in Figure 2. The first step of the antibacterial compound predictor is to prepare the benchmark dataset using screened active and inactive antibacterial compounds from the ChEMBL and the PubChem database. Then, the SVM, RF, and MLP methods were used to build models using the benchmark dataset. Using the parameter grid search and 5-fold cross-validation strategy, the optimal parameters of these three models were determined (Table 1, Supplementary Figures 1-3). After training, parameter optimization, and model evaluation, the optimal SVM, RF, and MLP models were established. The final antibacterial compound predictor includes the combination of the optimal three models. The integrated model was used to predict the antibacterial activity of FDA-approved small-molecule drugs from the DrugBank database.

\section{High performance of the SVM, RF, and MLP models}

The overall performance of the SVM, RF, and MLP models was quantified by multiple classification evaluation indicators including accuracy, precision, sensitivity, specificity, F1 score, AUC, and MSE (Table 1). The mean values of accuracy, precision, sensitivity, specificity, and F1 score of the three models at around 0.85 . The mean values of the AUC of these models were higher than 0.92 (ROC curves of these models shown in Supplementary Figure 4). The mean squared error of the three models is around 0.15 . These indicate that all three models showed high effectiveness in

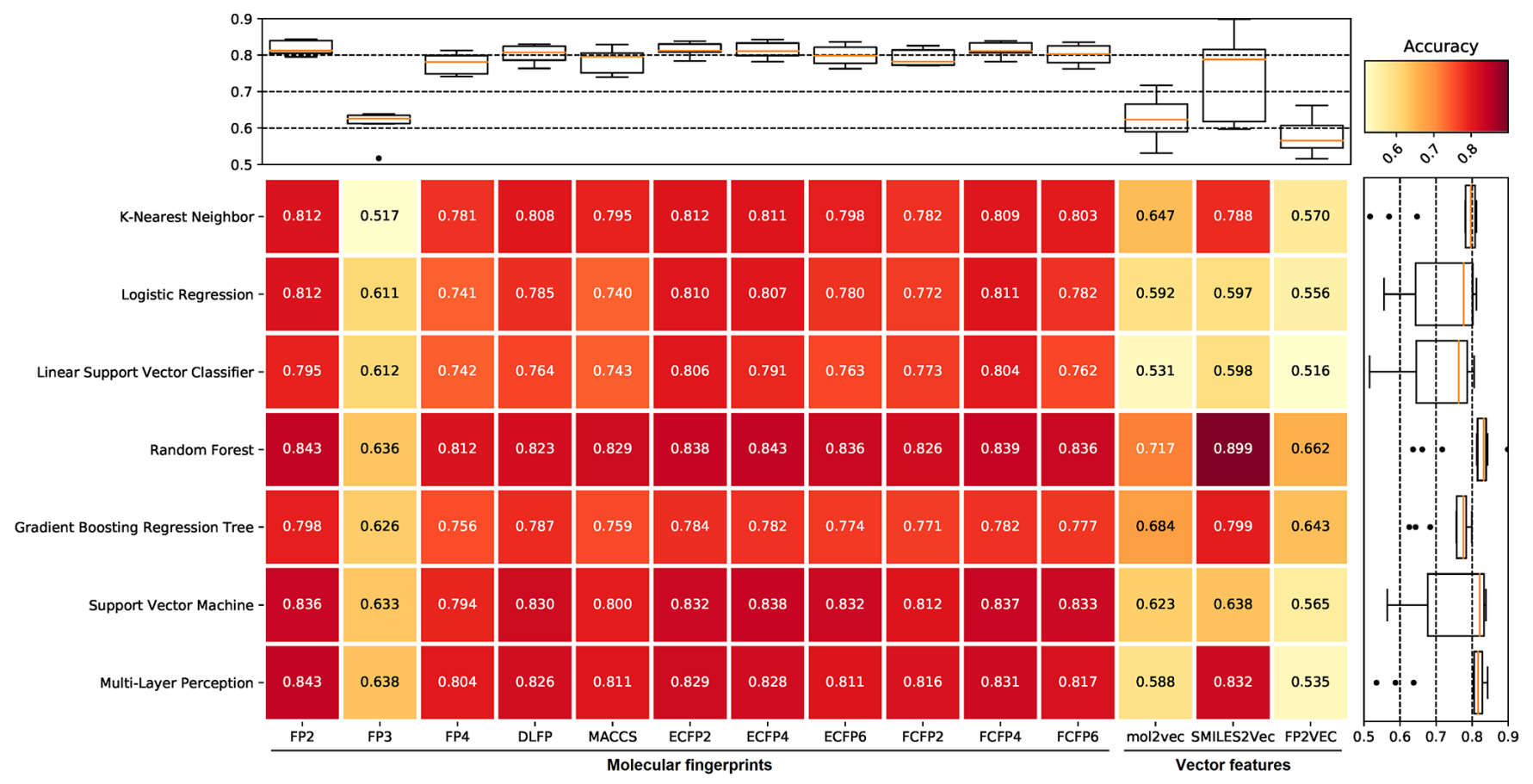

Figure 1. The prediction accuracy of different machine learning methods for benchmark datasets. The filtered datasets include one positive dataset and 10 negative datasets, therefore, each value in the figure is the average of 10 prediction accuracy. Compared with other machine learning methods, random forest (RF), support vector machine (SVM), and multi-layer perception (MLP) all show higher prediction accuracy. The benchmark dataset based on FP2 molecular fingerprints shows the highest prediction accuracy in the RF and MLP methods, and also shows high prediction accuracy in the SVM method among all molecular fingerprints. The accuracy fluctuates greatly among different machine learning methods in the benchmark dataset based on vector features. 
Table 1. Optimal parameters and prediction performance of different machine learning methods.

\begin{tabular}{lccc}
\hline & Support vector machine & Random forest & Multi-layer perception \\
\hline Optimal & gamma: 0.01 & n_estimators: 750 & hidden_layer_sizes: 512 \\
parameters & C:10 & & 0.0001 \\
Accuracy & $0.852 \pm 0.002$ & $0.849 \pm 0.004$ & $0.847 \pm 0.004$ \\
Precision & $0.854 \pm 0.004$ & $0.868 \pm 0.004$ & $0.850 \pm 0.007$ \\
Sensitivity & $0.850 \pm 0.004$ & $0.822 \pm 0.007$ & $0.845 \pm 0.003$ \\
Specificity & $0.854 \pm 0.005$ & $0.875 \pm 0.004$ & $0.850 \pm 0.009$ \\
F1 score & $0.852 \pm 0.002$ & $0.844 \pm 0.005$ & $0.847 \pm 0.003$ \\
AUC & $0.926 \pm 0.002$ & $0.932 \pm 0.002$ & $0.920 \pm 0.002$ \\
MSE & $0.148 \pm 0.002$ & $0.151 \pm 0.004$ & $0.153 \pm 0.004$ \\
\hline
\end{tabular}

Abbreviations: AUC: area under the curve; MSE: mean squared error. Parameters of predictive performance were displayed as mean \pm standard deviation.

antibacterial compounds prediction. Furthermore, the data showed that the standard deviations of these indicators are very small, suggesting that different negative datasets do not affect the overall performance of these models.

\section{Prediction of candidate antibacterial small-molecule drugs}

All approved small-molecule drugs in the DrugBank database were used to screen for potential antibacterial

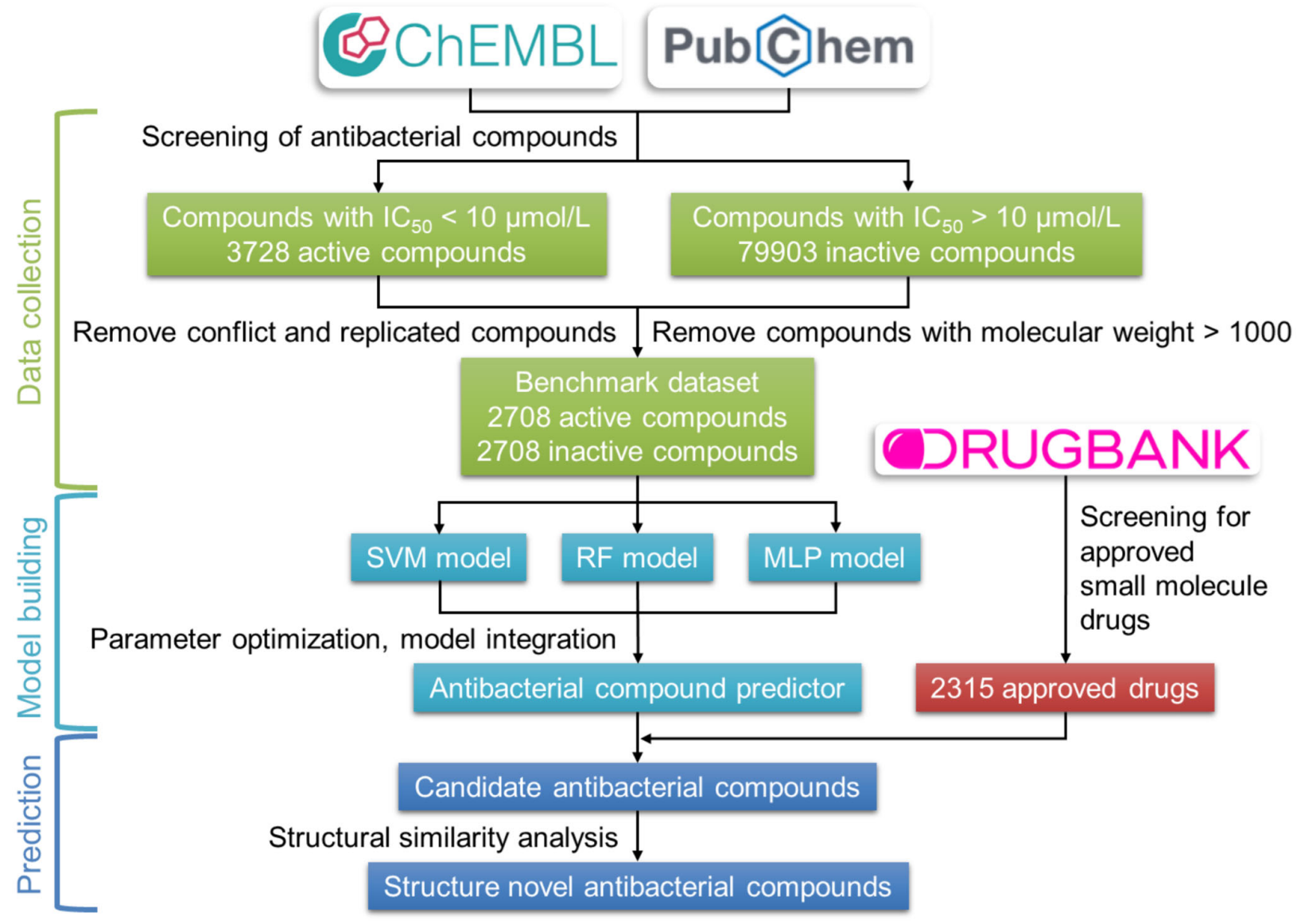

Figure 2. Flow chart of the construction of the antibacterial compound prediction model. The benchmark dataset was built using the active and inactive antibacterial compounds downloaded from the ChEMBL and the PubChem database. The combination of SVM, RF, and MLP methods was used to construct the antibacterial compounds predictor, which is used to predict the antibacterial activity of approved small-molecule drugs from the DrugBank database. 
compounds through the antibacterial compound predictor. The results showed that there are large differences in the number of drugs in isolated prediction intervals among the SVM, RF, and MLP models. There are more compounds in the probability intervals at both ends and fewer compounds in the middle intervals in the MLP model, whereas the distribution of predicted probabilities showed the opposite trend in the RF and SVM models (Figure 3A). There were 1482, 1539, and 1398 predicted active antibacterial drugs in the single SVM, RF, and MLP models. A total of 1090 drugs showed antibacterial activity shared by all three models

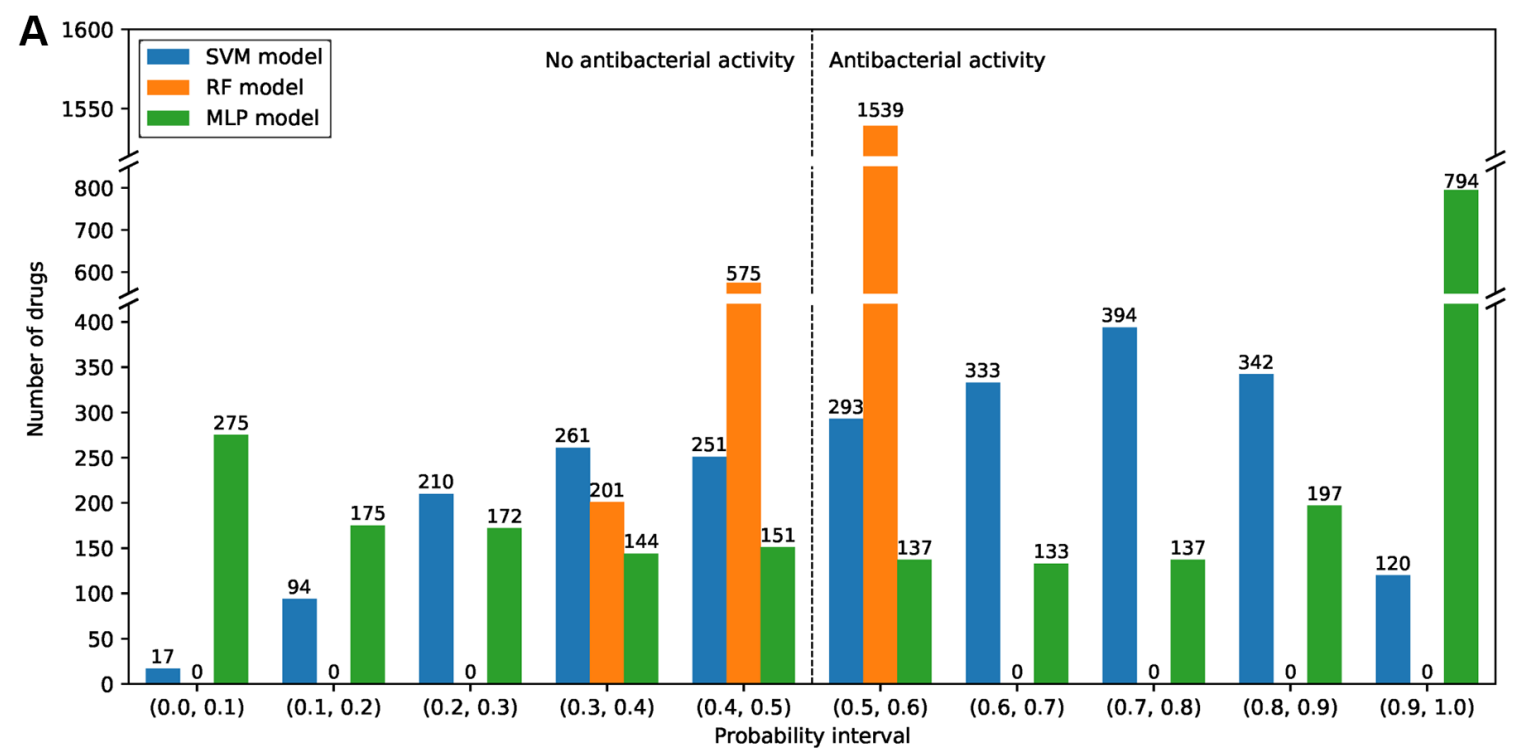

B

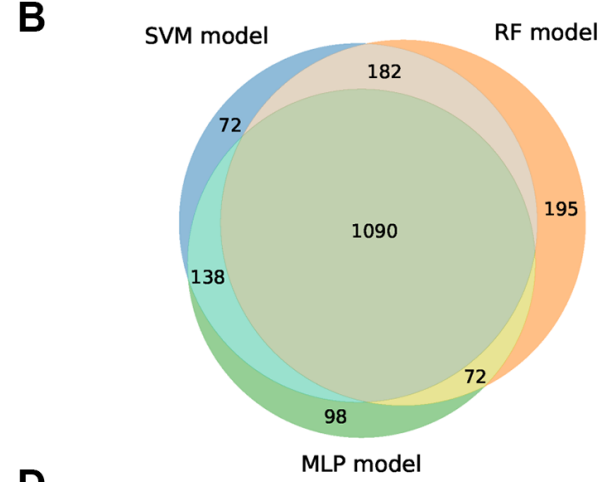

C
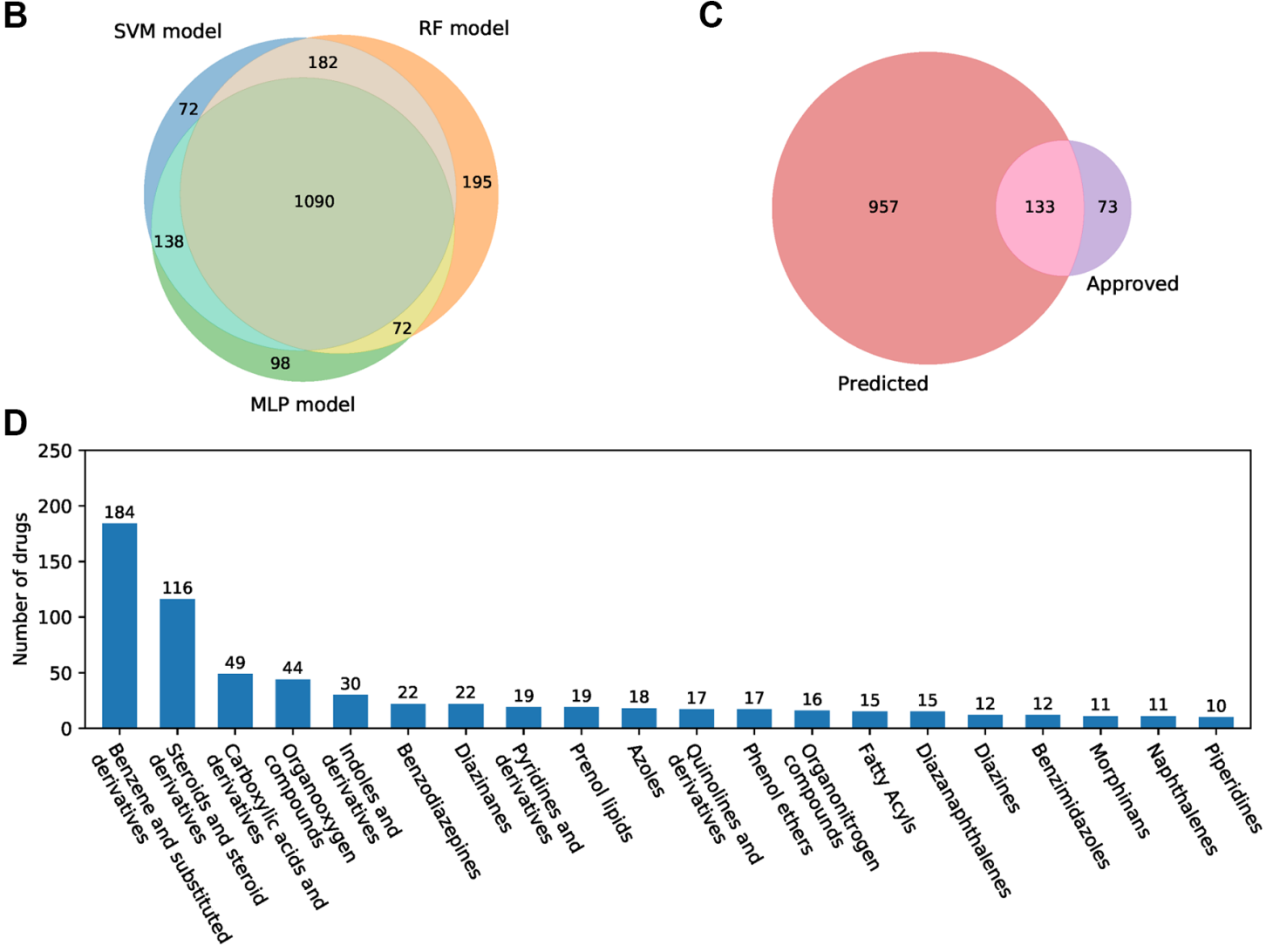

Figure 3. Antibacterial prediction results of approved small-molecule drugs. (A) The probability of predicted antibacterial activity for all small-molecule drugs in the SVM, RF, and MLP models. A drug with a probability value greater than 0.5 is considered an active antibacterial compound. (B) Venn diagram of the predicted antibacterial drugs in three machine learning models. (C) Venn diagram of the predicted antibacterial drugs and FDA-approved antibacterial drugs. (D) The top 20 categories of the 957 predicted novel antibacterial drugs. 
(Figure 3B). The single model and the combination of the two models predicted relatively more active antibacterial compounds, and there is more overlap with FDA-approved drugs (Supplementary Figure 5). Among the prediction results by the combination of the three models, 133 antibacterial drugs were FDA approved (Figure 3C). Our results suggested that both the single models and the combination of multiple models all showed excellent prediction performance (Supplementary Table 1). Furthermore, for the remaining 957 drugs, many of them belong to benzene and substituted derivatives (184 drugs) and steroids and steroid derivatives (116 drugs), few drugs belong to other categories (Figure 3D).

\section{Structural similarity of the predicted antibacterial drugs}

Molecular fingerprint similarity was calculated between the predicted and FDA-approved antibacterial drugs. The predicted antibacterial drugs that are not approved for marketing were defined as novel predicted antibacterial drugs. There were low overall similarities between approved antibacterial drugs and novel predicted antibacterial drugs (Supplementary Figure 6). 873 novel-predicted antibacterial drugs showed average similarities $\leq 0.2$ to all approved antibacterial drugs (Figure 4A). According to previous reports [32], we identified 8 representative core scaffolds from the
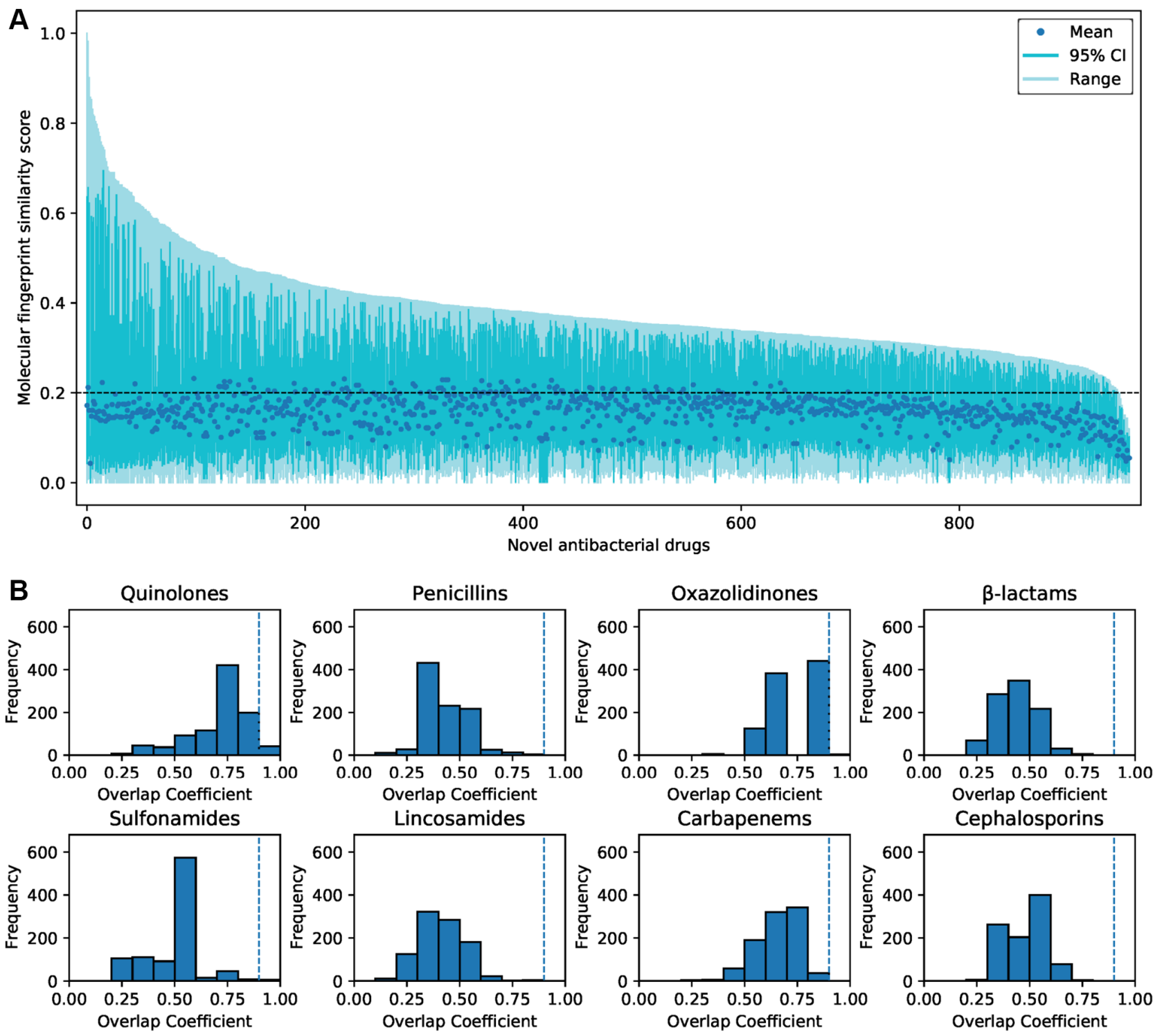

Figure 4. The similarity of the predicted antibacterial drugs and FDA-approved antibacterial drugs. (A) The molecular fingerprint similarity of 957 predicted novel antibacterial drugs and 206 FDA-approved antibacterial drugs. The average similarities between most of the predicted drugs and approved drugs were less than 0.2. (B) Substructure similarity between novel predicted antibacterial drugs and core scaffolds of approved antibacterial drugs. Compounds with an overlap coefficient higher than 0.9 are considered to have high substructure similarity. 
Table 2. The prediction results of 9 antibacterial drugs with low structural similarities.

\begin{tabular}{|c|c|c|c|c|c|}
\hline \multirow{2}{*}{ DrugBank ID } & \multirow{2}{*}{ Name } & \multicolumn{3}{|c|}{ Predicted probability } & \multirow{2}{*}{$\begin{array}{l}\text { Structural similarity } \\
(\text { mean }(\text { min-max }))^{1}\end{array}$} \\
\hline & & SVM & RF & MLP & \\
\hline DB00228 & Enflurane & 0.741 & 0.544 & 0.916 & $0.055(0.000-0.119)$ \\
\hline DB00531 & Cyclophosphamide & 0.571 & 0.518 & 0.902 & $0.086(0.010-0.150)$ \\
\hline DB00753 & Isoflurane & 0.698 & 0.536 & 0.980 & $0.055(0.000-0.120)$ \\
\hline DB00964 & Apraclonidine & 0.514 & 0.514 & 0.501 & $0.093(0.013-0.198)$ \\
\hline DB01028 & Methoxyflurane & 0.770 & 0.504 & 0.913 & $0.048(0.000-0.143)$ \\
\hline DB01057 & Echothiophate & 0.703 & 0.518 & 0.864 & $0.072(0.017-0.143)$ \\
\hline DB01181 & Ifosfamide & 0.589 & 0.515 & 0.888 & $0.095(0.010-0.172)$ \\
\hline DB01189 & Desflurane & 0.732 & 0.546 & 0.975 & $0.055(0.000-0.150)$ \\
\hline DB01236 & Sevoflurane & 0.538 & 0.517 & 0.934 & $0.060(0.000-0.162)$ \\
\hline
\end{tabular}

Abbreviations: SVM: support vector machine; RF: random forest; MLP: multi-layer perception. ${ }^{1}$ The structural similarities were calculated between the novel predicted antibacterial drugs and FDA-approved antibacterial drugs.

FDA-approved antibacterial drugs (Supplementary Table 2). 906 predicted compounds do not contain any core scaffold (Figure 4B). Only $51(5.3 \%)$ of the predicted compounds showed a high overlap coefficient with core scaffolds (Supplementary Table 3). These indicate that most of the predicted antibacterial drugs are structurally novel.

\section{Novel predicted antibacterial drugs}

There were 9 novel-predicted drugs with an average similarity less than 0.1 and a maximum similarity less than 0.2 to all approved antibacterial drugs, and these drugs all showed high predicted probability in SVM, RF, and MLP models (Table 2). Details of these 9 drugs are listed in Supplementary Table 4. Among these drugs, cyclophosphamide (DB00531) and ifosfamide (DB01181) are anticancer drugs that were used to treat a variety of hematological tumors and solid tumors. Apraclonidine (DB00964) is used to relieve postsurgical ocular hypertension. Echothiophate is used for the treatment of subacute or chronic angle-closure glaucoma. The other 5 drugs are mainly used in general anesthesia, such as enflurane (DB00228), isoflurane (DB00753), methoxyflurane (DB01028), desflurane (DB01189), and sevoflurane (DB01236). To explore the correlation between these drugs and aging, 307 human aging-related genes were downloaded from the Human Ageing Genomic Resources (HAGR, https://genomics.senescence.info/). We used SEA [33], HitPickV2 [34], and TargetNet [35] for target prediction of these 9 drugs, the union set of the three predictions were chosen as target genes for the query drug. The results showed that these drugs may target recognized aging genes (such as APP, AR, RELA, and SIRT1, Supplementary Figure 7).

\section{DISCUSSION}

Exploring the antibacterial activity of the approved drugs may be an effective way of screening new antibiotics. It is an effective approach by using machine learning methods to predict active antibacterial compounds $[26,28,36]$. The accuracy of the prediction model is affected by many factors, such as the quality of the benchmark datasets [37], the representative molecular characteristics of the compounds [16], the applicable machine learning models [9], and the optimized model parameters [38]. This study collected a large amount of experimental data on the antibacterial activity of compounds from the ChEMBL and PubChem databases. By comparing the prediction accuracy of multiple machine learning models on benchmark datasets constructed based on different molecular fingerprints, our results showed that the average prediction accuracy of SVM, RF, and MLP models are higher than other machine learning methods, and the FP2 molecular fingerprint is more representative than other fingerprints. Therefore, it is reasonable to construct the antibacterial compound predictor by building the benchmark datasets by calculating the FP2 molecular fingerprint of the compounds and combining the RF, SVM, and MLP models. However, the model constructed in this study did not achieve the desired prediction performance (only 133 of the 206 FDA-approved antibacterial drugs have been successfully predicted). This is probably because the benchmark datasets collected data from multiple sources and require a more effective data integration strategy. Furthermore, it is worth noting that parameter optimization can only slightly improve (approximately 1\%) the prediction accuracy of the different machine learning models. 
Through structural similarity analysis of the predicted active antibacterial drugs, we screened 9 drugs with novel structures. Apraclonidine is mainly used for the prevention and treatment of post-surgical intraocular pressure (IOP) elevation, and it is also indicated for the short-term adjunctive treatment of glaucoma [39]. Echothiophate is used in the treatment of subacute or chronic angle-closure glaucoma and some cases it is also used as accommodative esotropia [40]. Cyclophosphamide and ifosfamide are widely used broad-spectrum anticancer drugs [41, 42]. Studies showed that cyclophosphamide can inhibit bacterial translocation of the gastrointestinal tract [43] and reduce the abundance of lactobacilli and enterococci [44] in mice. Desflurane, enflurane, isoflurane, methoxyflurane, and sevoflurane are widely used volatile anesthetics $[45,46]$, most of these anesthetics have demonstrated antibacterial properties in vitro [4750]. An early in vitro experiment showed that methoxyflurane and isoflurane exhibited excellent antibacterial activity, while enflurane had less effect on a few pathogens [48]. The resistance experiment to a variety of bacteria showed that isoflurane has higher antibacterial activity than sevoflurane [49]. Based on these reports, the antibacterial compound screening method used in this study is credible.

There are still many difficulties in the discovery of antibacterial compounds in silico. Firstly, the prediction accuracy is affected by the size and quality of the benchmark dataset. The definition of the active or inactive antibacterial compounds in this study is based on the in vitro experimental data. However, most of the screened active antibacterial compounds have not yet entered clinical trials, the human safety and clinical effectiveness of these compounds are still unclear [51]. Then, the compounds in this study were characterized by molecular fingerprints, whereas this method cannot reflect the complete structural features of given compounds and is not suitable for macromolecular compounds [16]. Next, machine learning models need further optimization. The prediction accuracy of the SVM, RF, and MLP models in this study is around 0.85 , optimizing these models may be able to obtain higher prediction accuracy. Lastly, considering that compounds may produce different types of molecules during the metabolic process, computational simulation of the drug metabolic process [52] in humans will make the predictions more convincing.

The development of new and highly effective antibiotics can alleviate the crisis of bacterial infections, extend human lifespan, and reduce the incidence of infectious diseases in the elderly. This study provides a new insight for predicting antibacterial compounds with novel structures by using approved drugs. The existing approach could be extended by different augmentation methods (such as compound augmentation by graph or molecular description) with different machine learning state-of-the-art methods such as deep-learning methods. There are still many challenges and opportunities in using machine learning to predict antibacterial compounds. With the development of big data technology, the continuous optimization of machine learning models and algorithms, and the discovery of more antibacterial active compounds and drugs, it is foreseeable that the prediction of antibacterial compounds in the future will achieve higher accuracy and credibility.

\section{MATERIALS AND METHODS}

\section{Antibacterial compounds collection}

Compounds that performed antimicrobial activity tests were collected from ChEMBLdb (version 25, https://www.ebi.ac.uk/chembl/) and PubChem (https://pubchem.ncbi.nlm.nih.gov/) databases. A total of 83768 compounds were obtained, 8001 of these compounds have a clear IC50 value, and others only have an inactive label. The IC50 cutoff value of antibacterial activity was defined by curve fitting the IC50 values of all compounds. Compounds with IC50 less than $10 \mu \mathrm{mol} / \mathrm{L}$ were generally considered as active antibacterial compounds [53-57], the curve fitting results also suggest that this cutoff is reasonable (Supplementary Figure 8). Based on the curve fitting results, compounds with IC50 higher than $10 \mu \mathrm{mol} / \mathrm{L}$ were considered inactive antibacterial compounds. Pybel, a python wrapper of OpenBabel $[58,59]$ was used to access the SMILES string of compounds and calculate molecular fingerprint which represents the presence or absence of particular substructures in the molecule. Multiple types of molecular fingerprints of all compounds were calculated. Benchmark datasets were built based on the following steps: (1) remove duplicate compounds; (2) remove compounds with a molecular weight greater than 1000; (3) remove compounds with molecular fingerprint similarities higher than 0.9 between the active and inactive antibacterial compounds. Finally, we got a positive dataset including 2708 active antibacterial compounds and a negative dataset including 78620 inactive antibacterial compounds. All active antibacterial compounds have IC50 values whereas only 1893 inactive antibacterial compounds have IC50 values.

\section{Construction of the benchmark dataset}

There is a large difference in the number of compounds between the positive and negative datasets. The positive 
dataset contains 2708 active antibacterial compounds. To balance the number of compounds between the positive and negative datasets, the filtered negative dataset contains 1893 inactive antibacterial compounds with IC50 values, the remaining quantity difference was randomly selected from the inactive antibacterial compounds only with an inactive label. Considering the uncertainty of random selection, we repeated 10 times for negative dataset extract. Therefore, the filtered datasets including one positive dataset and 10 negative datasets, each negative dataset are combined with the positive data set for subsequent analysis. Next, the molecular fingerprint is calculated for the positive dataset and all repeated negative datasets. The following types of molecular fingerprints were calculated including FP2, FP3, FP4, DLFP, MACCS, ECFP2, ECFP4, ECFP6, FCFP2, FCFP4, and FCFP6. Several start-of-the-art chemoinformatics approaches were also calculated such as mol2vec [60], SMILES2Vec [61], and FP2VEC [62]. The features of each compound were presented by the binary bits of the different types of molecular fingerprints or vectors and these features were used for machine learning modeling (Supplementary Table 5). All these benchmark datasets were used for the preliminary screening of applicable machine learning models.

\section{Parameter selection of the SVM, RF, and MLP models}

The SVM, RF, and MLP models for antibacterial compounds prediction were built using the svm, ensemble, and neural_network module in the scikitlearn Python library (version: 0.20.0, https://scikitlearn.org/stable/). A parameter grid search strategy was used to choose the optimal parameter "gamma" for the kernel function and regularization parameter " $\mathrm{C}$ " for the SVM model, the optimal number of trees (parameter "n_estimators") for the RF model, and the optimal hidden layer sizes and alpha for the MLP model. The other parameters of the above three models use default values. The benchmark dataset was randomly split into the training and validation set (accounting for 80\%) and the test set (accounting for 20\%) using the train test_split function in the scikit-learn. The 5-fold cross-validation method was used to evaluate the generalization performance of the model with specified parameters in the training and validation set. The crossvalidation accuracy was calculated for model evaluation. After cross-validation, a temporary model was built using the training and validation set and calculated the area under the curve (AUC) for the receiver operating characteristic (ROC) curve in the test set. Considering that there may be similar compounds in the split datasets, dataset split and cross-validation were repeated 10 times, which may reduce the impact of similar compounds on the prediction performance of these models. For each given parameter, the mean cross-validation accuracy and mean AUC was calculated. The optimal model was selected by comparing the maximum mean cross-validation accuracy under different parameters. If there were multiple models with the same mean accuracy, the model with the maximum AUC was considered to be the optimal model.

\section{Performance evaluation}

The optimal SVM, RF, and MLP models were used for performance evaluation. The confusion matrix was calculated using the results of the optimal crossvalidation test. The true positive (TP) indicates the number of correctly predicted active antibacterial compounds, the true negative (TN) indicates the number of correctly predicted inactive antibacterial compounds, the false positive (FP) indicates the number of inactive antibacterial compounds predicted as active antibacterial compounds, and the false negative (FN) indicates the number of active antibacterial compounds predicted as inactive antibacterial compounds. We calculated the following quality indices: accuracy $=(\mathrm{TP}$ $+\mathrm{TN}) /(\mathrm{TP}+\mathrm{TN}+\mathrm{FP}+\mathrm{FN})$, precision $=\mathrm{TP} /(\mathrm{TP}+\mathrm{FP})$, sensitivity $=\mathrm{TP} /(\mathrm{TP}+\mathrm{FN})$, specificity $=\mathrm{TN} /(\mathrm{TN}+\mathrm{FP})$, and $\mathrm{F} 1$ score $=2 \times \mathrm{TP} /(2 \times \mathrm{TP}+\mathrm{FP}+\mathrm{FN})$. Mean squared error (MSE) was calculated for all three models. Because the filtered datasets include one positive dataset and 10 negative datasets, the average of 10 calculations of these quality indices and AUC were used to evaluate the SVM, RF, and MLP model performance. A model with high scores $(\geq 0.8)$ of accuracy, precision, F1 score, and AUC was considered to be an effective model.

\section{Antibacterial small-molecule drugs prediction}

The final SVM, RF, and MLP models were built using the benchmark dataset with the optimal parameters. All these three models were used to predict antibacterial activity for approved small-molecule drugs. We compared the prediction performance of a single model and a combination of different models. The candidate antibacterial drugs were defined as the drugs that showed antibacterial activity in all the SVM, RF, and MLP models. Drug information was acquired from the DrugBank database (https://www.drugbank.ca/) [63]. We first filtered the drugs with approved status but not withdrawn yet, then removed the drugs with molecular weight $\geq 1000$. Finally, 2315 approved small-molecule drugs were screened to perform antibacterial activity prediction. The predicted active antibacterial drugs excluding FDA-approved antibacterial drugs were defined as novel antibacterial drugs. 


\section{Structural similarity analysis}

FP2 molecular fingerprint similarity was calculated among all novel antibacterial drugs and FDA-approved antibacterial drugs. The overlap between fingerprints is quantified as a measure of molecular similarity using the Tanimoto coefficient (Tc). The predicted drugs with average and maximum molecular fingerprint similarity less than 0.1 and 0.2 were considered to be structurally novel. Furthermore, previous literature reported several core scaffolds shared by most antibacterial compounds [32]. The flexible maximum common substructure algorithms in the fmcsR package [64] in $\mathrm{R}$ were used to identify whether the core scaffolds exist in the predicted antibacterial drugs.

\section{AUTHOR CONTRIBUTIONS}

WXL, DL, and SXD designed the study. WXL, GHL, and SXD collected the data. WXL, XT, PPY, YZ, and DGG designed the method and analyzed the data. WXL, JHL, and SXD wrote the manuscript. All authors read and approved the submitted version.

\section{CONFLICTS OF INTEREST}

The authors declare no conflicts of interest related to this study.

\section{FUNDING}

This work was supported by the Start-up Fund of Kunming University of Science and Technology (Grant No. KKZ3201927005 for SXD), the Yunnan Fundamental Research Projects (Grant No. 2019FB050 for SXD), the Start-up Fund of Southern Medical University (Grant No. G820282016 for DGG), and the National Natural Science Foundation of China (Grant Nos. 31501080 and 32070676 for DGG). The funding bodies had no role in the design of the study, collection, analysis, and interpretation of data, or in writing the manuscript.

\section{REFERENCES}

1. Allen JC, Toapanta FR, Chen W, Tennant SM. Understanding immunosenescence and its impact on vaccination of older adults. Vaccine. 2020; 38:8264-72.

https://doi.org/10.1016/j.vaccine.2020.11.002 PMID:33229108

2. Brown GC. The endotoxin hypothesis of neurodegeneration. J Neuroinflammation. 2019; 16:180. https://doi.org/10.1186/s12974-019-1564-7 PMID: $\underline{31519175}$
3. Panza F, Lozupone $M$, Solfrizzi V, Watling $M$, Imbimbo BP. Time to test antibacterial therapy in Alzheimer's disease. Brain. 2019; 142:2905-29.

https://doi.org/10.1093/brain/awz244

PMID:31532495

4. Tacconelli E, Carrara E, Savoldi A, Harbarth S, Mendelson M, Monnet DL, Pulcini C, Kahlmeter G, Kluytmans J, Carmeli Y, Ouellette M, Outterson K, Patel $\mathrm{J}$, et al, and WHO Pathogens Priority List Working Group. Discovery, research, and development of new antibiotics: the WHO priority list of antibiotic-resistant bacteria and tuberculosis. Lancet Infect Dis. 2018; 18:318-27.

https://doi.org/10.1016/S1473-3099(17)30753-3 PMID:29276051

5. Jackson N, Czaplewski L, Piddock LJV. Discovery and development of new antibacterial drugs: learning from experience? J Antimicrob Chemother. 2018; 73:1452-9.

https://doi.org/10.1093/jac/dky019

PMID:29438542

6. Pereira DA, Williams JA. Origin and evolution of high throughput screening. Br J Pharmacol. 2007; 152:53-61. https://doi.org/10.1038/sj.bjp.0707373 PMID: 17603542

7. Mishra KP, Ganju L, Sairam M, Banerjee PK, Sawhney RC. A review of high throughput technology for the screening of natural products. Biomed Pharmacother. 2008; 62:94-8.

https://doi.org/10.1016/i.biopha.2007.06.012 PMID: 17692498

8. Tommasi R, Brown DG, Walkup GK, Manchester JI, Miller AA. ESKAPEing the labyrinth of antibacterial discovery. Nat Rev Drug Discov. 2015; 14:529-42.

https://doi.org/10.1038/nrd4572 PMID:26139286

9. Durrant JD, Amaro RE. Machine-learning techniques applied to antibacterial drug discovery. Chem Biol Drug Des. 2015; 85:14-21. https://doi.org/10.1111/cbdd.12423 PMID:25521642

10. Nicolaou CA, Brown N. Multi-objective optimization methods in drug design. Drug Discov Today Technol. 2013; 10:e427-35. https://doi.org/10.1016/i.ddtec.2013.02.001 PMID:24050140

11. Cummins DJ, Bell MA. Integrating Everything: The Molecule Selection Toolkit, a System for Compound Prioritization in Drug Discovery. J Med Chem. 2016; 59:6999-7010. https://doi.org/10.1021/acs.jmedchem.5b01338 PMID:26950497 
12. Halder AK, Dias Soeiro Cordeiro MN. QSAR-Co-X: an open source toolkit for multitarget QSAR modelling. J Cheminform. 2021; 13:29.

https://doi.org/10.1186/s13321-021-00508-0 PMID:33858509

13. Sánchez-Rodríguez A, Pérez-Castillo $Y$, Schürer SC, Nicolotti O, Mangiatordi GF, Borges F, Cordeiro MND, Tejera E, Medina-Franco JL, Cruz-Monteagudo M. From flamingo dance to (desirable) drug discovery: a nature-inspired approach. Drug Discov Today. 2017; 22:1489-502.

https://doi.org/10.1016/i.drudis.2017.05.008 PMID:28624633

14. Speck-Planche A, Cordeiro MN. Simultaneous modeling of antimycobacterial activities and ADMET profiles: a chemoinformatic approach to medicinal chemistry. Curr Top Med Chem. 2013; 13:1656-65. https://doi.org/10.2174/15680266113139990116 PMID:23889052

15. Cereto-Massagué A, Ojeda MJ, Valls C, Mulero M, Garcia-Vallvé S, Pujadas G. Molecular fingerprint similarity search in virtual screening. Methods. 2015; 71:58-63.

https://doi.org/10.1016/j.ymeth.2014.08.005 PMID:25132639

16. Muegge I, Mukherjee P. An overview of molecular fingerprint similarity search in virtual screening. Expert Opin Drug Discov. 2016; 11:137-48. https://doi.org/10.1517/17460441.2016.1117070 PMID:26558489

17. Vamathevan J, Clark D, Czodrowski P, Dunham I, Ferran E, Lee G, Li B, Madabhushi A, Shah P, Spitzer $M$, Zhao $S$. Applications of machine learning in drug discovery and development. Nat Rev Drug Discov. 2019; 18:463-77.

https://doi.org/10.1038/s41573-019-0024-5

PMID: $\underline{30976107}$

18. Chen $X$, Yan CC, Zhang $X$, Zhang $X$, Dai F, Yin J, Zhang $Y$. Drug-target interaction prediction: databases, web servers and computational models. Brief Bioinform. 2016; 17:696-712. https://doi.org/10.1093/bib/bbv066 PMID:26283676

19. D'Souza S, Prema KV, Balaji S. Machine learning models for drug-target interactions: current knowledge and future directions. Drug Discov Today. 2020; 25:748-56.

https://doi.org/10.1016/j.drudis.2020.03.003 PMID: $\underline{32171918}$

20. Walters WP, Barzilay R. Applications of Deep Learning in Molecule Generation and Molecular Property Prediction. Acc Chem Res. 2021; 54:263-70. https://doi.org/10.1021/acs.accounts.0c00699 PMID:33370107

21. Ekins S, Puhl AC, Zorn KM, Lane TR, Russo DP, Klein JJ, Hickey AJ, Clark AM. Exploiting machine learning for end-to-end drug discovery and development. Nat Mater. 2019; 18:435-41. https://doi.org/10.1038/s41563-019-0338-z PMID: $\underline{1000803}$

22. Lata S, Sharma BK, Raghava GP. Analysis and prediction of antibacterial peptides. BMC Bioinformatics. 2007; 8:263. https://doi.org/10.1186/1471-2105-8-263 PMID:17645800

23. Khosravian M, Faramarzi FK, Beigi MM, Behbahani M, Mohabatkar H. Predicting antibacterial peptides by the concept of Chou's pseudo-amino acid composition and machine learning methods. Protein Pept Lett. 2013; 20:180-6. https://doi.org/10.2174/092986613804725307 PMID:22894156

24. Zoffmann S, Vercruysse M, Benmansour F, Maunz A, Wolf L, Blum Marti R, Heckel T, Ding H, Truong HH, Prummer M, Schmucki R, Mason CS, Bradley K, et al. Machine learning-powered antibiotics phenotypic drug discovery. Sci Rep. 2019; 9:5013.

https://doi.org/10.1038/s41598-019-39387-9 PMID:30899034

25. Rahman SF, Olm MR, Morowitz MJ, Banfield JF. Machine Learning Leveraging Genomes from Metagenomes Identifies Influential Antibiotic Resistance Genes in the Infant Gut Microbiome. mSystems. 2018; 3:e00123-17. https://doi.org/10.1128/mSystems.00123-17 PMID:29359195

26. Stokes JM, Yang K, Swanson K, Jin W, Cubillos-Ruiz A, Donghia NM, MacNair CR, French S, Carfrae LA, Bloom-Ackermann Z, Tran VM, Chiappino-Pepe A, Badran AH, et al. A Deep Learning Approach to Antibiotic Discovery. Cell. 2020; 180:688-702.e13. https://doi.org/10.1016/i.cell.2020.01.021 PMID: $\underline{32084340}$

27. Yang XG, Chen D, Wang M, Xue Y, Chen YZ. Prediction of antibacterial compounds by machine learning approaches. J Comput Chem. 2009; 30:1202-11. https://doi.org/10.1002/icc.21148 PMID:18988254

28. Kavvas ES, Catoiu E, Mih N, Yurkovich JT, Seif Y, Dillon $\mathrm{N}$, Heckmann D, Anand A, Yang L, Nizet V, Monk JM, Palsson BO. Machine learning and structural analysis of Mycobacterium tuberculosis pan-genome identifies genetic signatures of antibiotic resistance. Nat Commun. 2018; 9:4306. 
https://doi.org/10.1038/s41467-018-06634-y PMID: $\underline{30333483}$

29. Niehaus KE, Walker TM, Crook DW, Peto TEA, Clifton DA. Machine learning for the prediction of antibacterial susceptibility in Mycobacterium tuberculosis. IEEE-EMBS International Conference on Biomedical and Health Informatics (BHI). 2014; 61821.

https://doi.org/10.1109/BHI.2014.6864440

30. Hefti FF. Requirements for a lead compound to become a clinical candidate. BMC Neurosci. 2008 (Suppl 3); 9:S7.

https://doi.org/10.1186/1471-2202-9-S3-S7 PMID:19091004

31. Savoia D. New Antimicrobial Approaches: Reuse of Old Drugs. Curr Drug Targets. 2016; 17:731-8. https://doi.org/10.2174/13894501166661508061241 $\underline{10}$ PMID:26245476

32. Fair RJ, Tor Y. Antibiotics and bacterial resistance in the 21st century. Perspect Medicin Chem. 2014; 6:25-64.

https://doi.org/10.4137/PMC.S14459

PMID:25232278

33. Keiser MJ, Roth BL, Armbruster BN, Ernsberger $\mathrm{P}$, Irwin JJ, Shoichet BK. Relating protein pharmacology by ligand chemistry. Nat Biotechnol. 2007; 25:197206.

https://doi.org/10.1038/nbt1284

PMID: 17287757

34. Hamad S, Adornetto G, Naveja JJ, Chavan Ravindranath A, Raffler J, Campillos M. HitPickV2: a web server to predict targets of chemical compounds. Bioinformatics. 2019; 35:1239-40.

https://doi.org/10.1093/bioinformatics/bty759 PMID:30169615

35. Yao ZJ, Dong J, Che YJ, Zhu MF, Wen M, Wang NN, Wang S, Lu AP, Cao DS. TargetNet: a web service for predicting potential drug-target interaction profiling via multi-target SAR models. J Comput Aided Mol Des. 2016; 30:413-24.

https://doi.org/10.1007/s10822-016-9915-2

PMID:27167132

36. Yang JH, Wright $S N$, Hamblin $M$, McCloskey $D$, Alcantar MA, Schrübbers L, Lopatkin AJ, Satish S, Nili A, Palsson BO, Walker GC, Collins JJ. A White-Box Machine Learning Approach for Revealing Antibiotic Mechanisms of Action. Cell. 2019; 177:1649-61.e9. https://doi.org/10.1016/i.cell.2019.04.016 PMID:31080069

37. Beltran JA, Aguilera-Mendoza L, Brizuela CA. Optimal selection of molecular descriptors for antimicrobial peptides classification: an evolutionary feature weighting approach. BMC Genomics. 2018; 19:672.

https://doi.org/10.1186/s12864-018-5030-1

PMID:30255784

38. Díaz I. Machine learning in the estimation of causal effects: targeted minimum loss-based estimation and double/debiased machine learning. Biostatistics. 2020; 21:353-8.

https://doi.org/10.1093/biostatistics/kxz042

PMID: $\underline{31742333}$

39. Harasymowycz P, Royer C, Cui AX, Barbeau M, JobinGervais K, Mathurin K, Lachaine J, Beauchemin C. Short-term efficacy of latanoprostene bunod for the treatment of open-angle glaucoma and ocular hypertension: a systematic literature review and a network meta-analysis. Br J Ophthalmol. 2021. [Epub ahead of print].

https://doi.org/10.1136/bjophthalmol-2020-317262

PMID:33397657

40. Kini MM, Dahl AA, Roberts CR, Lehwalder LW, Grant WM. Echothiophate, pilocarpine, and open-angle glaucoma. Arch Ophthalmol. 1973; 89:190-2. https://doi.org/10.1001/archopht.1973.01000040192 $\underline{005}$

PMID:4691318

41. Emadi A, Jones RJ, Brodsky RA. Cyclophosphamide and cancer: golden anniversary. Nat Rev Clin Oncol. 2009; 6:638-47.

https://doi.org/10.1038/nrclinonc.2009.146 PMID:19786984

42. Matz EL, Hsieh MH. Review of Advances in Uroprotective Agents for Cyclophosphamide- and Ifosfamide-induced Hemorrhagic Cystitis. Urology. 2017; 100:16-9.

https://doi.org/10.1016/j.urology.2016.07.030 PMID:27566144

43. Suzuki T, Itoh K, Hagiwara T, Nakayama H, Honjyo K, Hirota $Y$, Kaneko T, Suzuki $H$. Inhibition of bacterial translocation from the gastrointestinal tract of mice injected with cyclophosphamide. Curr Microbiol. 1996; 33:78-83.

https://doi.org/10.1007/s002849900078 PMID:8662176

44. Alexander JL, Wilson ID, Teare J, Marchesi JR, Nicholson JK, Kinross JM. Gut microbiota modulation of chemotherapy efficacy and toxicity. Nat Rev Gastroenterol Hepatol. 2017; 14:356-65.

https://doi.org/10.1038/nrgastro.2017.20 PMID:28270698

45. Kharasch ED, Thummel KE. Identification of cytochrome P450 2E1 as the predominant enzyme catalyzing human liver microsomal defluorination of 
sevoflurane, isoflurane, and methoxyflurane. Anesthesiology. 1993; 79:795-807.

https://doi.org/10.1097/00000542-199310000-00023 PMID: 8214760

46. Terrell RC, Warner DS. The invention and development of enflurane, isoflurane, sevoflurane, and desflurane. Anesthesiology. 2008; 108:531-3.

https://doi.org/10.1097/ALN.0b013e31816499cc PMID:18292690

47. Horton JN, Sussman M, Mushin WW. The antibacterial action of anaesthetic vapours. $\mathrm{Br} \mathrm{J}$ Anaesth. 1970; 42:483-7.

https://doi.org/10.1093/bja/42.6.483

PMID:4912643

48. Giorgi A, Parodi F, Piacenza G, Mantellini E, Salio M, Cremonte LG, Grosso E. [Antibacterial and antifungal activity of isoflurane and common anesthetic gases]. Minerva Med. 1986; 77:2007-10.

PMID:3534634

49. Martínez-Serrano M, Gerónimo-Pardo M, MartínezMonsalve A, Crespo-Sánchez MD. Antibacterial effect of sevoflurane and isoflurane. Rev Esp Quimioter. 2017; 30:84-9.

PMID:28198170

50. Imbernón-Moya A, Ortiz-de Frutos FJ, SanjuanAlvarez M, Portero-Sanchez I, Merinero-Palomares R, Alcazar V. Topical sevoflurane for chronic venous ulcers infected by multi-drug-resistant organisms. Int Wound J. 2017; 14:1388-90.

https://doi.org/10.1111/iwj.12794

PMID:28736974

51. Pogodin PV, Lagunin AA, Rudik AV, Druzhilovskiy DS, Filimonov DA, Poroikov VV. AntiBac-Pred: A Web Application for Predicting Antibacterial Activity of Chemical Compounds. J Chem Inf Model. 2019; 59:4513-8.

https://doi.org/10.1021/acs.jcim.9b00436 PMID:31661960

52. Roy H, Nandi S. In-Silico Modeling in Drug Metabolism and Interaction: Current Strategies of Lead Discovery. Curr Pharm Des. 2019; 25:3292-305. https://doi.org/10.2174/13816128256661909031559 $\underline{35}$ PMID:31481001

53. Choi KE, Balupuri A, Kang NS. The Study on the hERG Blocker Prediction Using Chemical Fingerprint Analysis. Molecules. 2020; 25:2615.

https://doi.org/10.3390/molecules25112615 PMID:32512802

54. Lind AP, Anderson PC. Predicting drug activity against cancer cells by random forest models based on minimal genomic information and chemical properties. PLoS One. 2019; 14:e0219774. https://doi.org/10.1371/journal.pone.0219774 PMID:31295321

55. Deng YH, Wang NN, Zou ZX, Zhang L, Xu KP, Chen AF, Cao DS, Tan GS. Multi-Target Screening and Experimental Validation of Natural Products from Selaginella Plants against Alzheimer's Disease. Front Pharmacol. 2017; 8:539.

https://doi.org/10.3389/fphar.2017.00539

PMID:28890698

56. Rifaioglu AS, Nalbat E, Atalay V, Martin MJ, CetinAtalay R, Doğan T. DEEPScreen: high performance drug-target interaction prediction with convolutional neural networks using 2-D structural compound representations. Chem Sci. 2020; 11:2531-57.

https://doi.org/10.1039/c9sc03414e

PMID:33209251

57. Li GH, Huang JF. CDRUG: a web server for predicting anticancer activity of chemical compounds. Bioinformatics. 2012; 28:3334-5.

https://doi.org/10.1093/bioinformatics/bts625 PMID:23080119

58. O'Boyle NM, Morley C, Hutchison GR. Pybel: a Python wrapper for the OpenBabel cheminformatics toolkit. Chem Cent J. 2008; 2:5.

https://doi.org/10.1186/1752-153x-2-5

PMID:18328109

59. O'Boyle NM, Banck M, James CA, Morley C, Vandermeersch T, Hutchison GR. Open Babel: An open chemical toolbox. J Cheminform. 2011; 3:33.

https://doi.org/10.1186/1758-2946-3-33

PMID:21982300

60. Jaeger S, Fulle S, Turk S. Mol2vec: Unsupervised Machine Learning Approach with Chemical Intuition. J Chem Inf Model. 2018; 58:27-35.

https://doi.org/10.1021/acs.jcim.7b00616 PMID:29268609

61. Öztürk H, Ozkirimli E, Özgür A. A novel methodology on distributed representations of proteins using their interacting ligands. Bioinformatics. 2018; 34:i295-303. https://doi.org/10.1093/bioinformatics/bty287 PMID:29949957

62. Jeon W, Kim D. FP2VEC: a new molecular featurizer for learning molecular properties. Bioinformatics. 2019; 35:4979-85. https://doi.org/10.1093/bioinformatics/btz307 PMID: $\underline{1070725}$

63. Wishart DS, Knox C, Guo AC, Shrivastava S, Hassanali M, Stothard P, Chang Z, Woolsey J. DrugBank: a comprehensive resource for in silico drug discovery and exploration. Nucleic Acids Res. 2006; 34:D668-72. https://doi.org/10.1093/nar/gkj067 PMID:16381955 
64. Wang Y, Backman TW, Horan K, Girke T. fmcsR: mismatch tolerant maximum common substructure searching in R. Bioinformatics. 2013; 29:2792-4.

https://doi.org/10.1093/bioinformatics/btt475

PMID:23962615 


\section{SUPPLEMENTARY MATERIALS}

\section{Supplementary Figures}
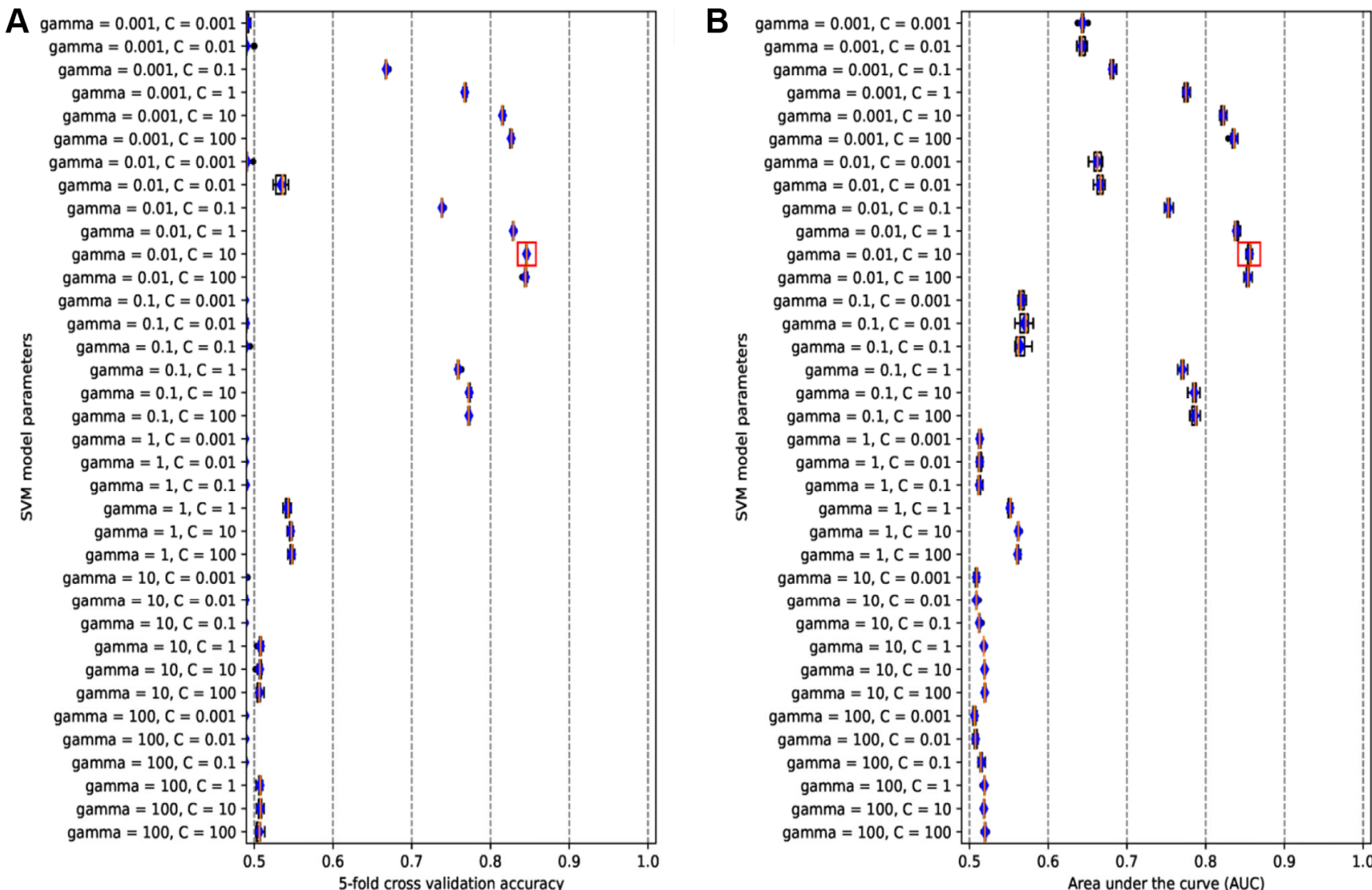

Supplementary Figure 1. Parameter optimization of the support vector machine (SVM) model. The optimal parameter "gamma" and " $C$ " was determined according to the maximum 5-fold cross-validation accuracy (A) and the maximum area under the curve (AUC) (B). The red box indicates the maximum 5-fold cross-validation accuracy or AUC.
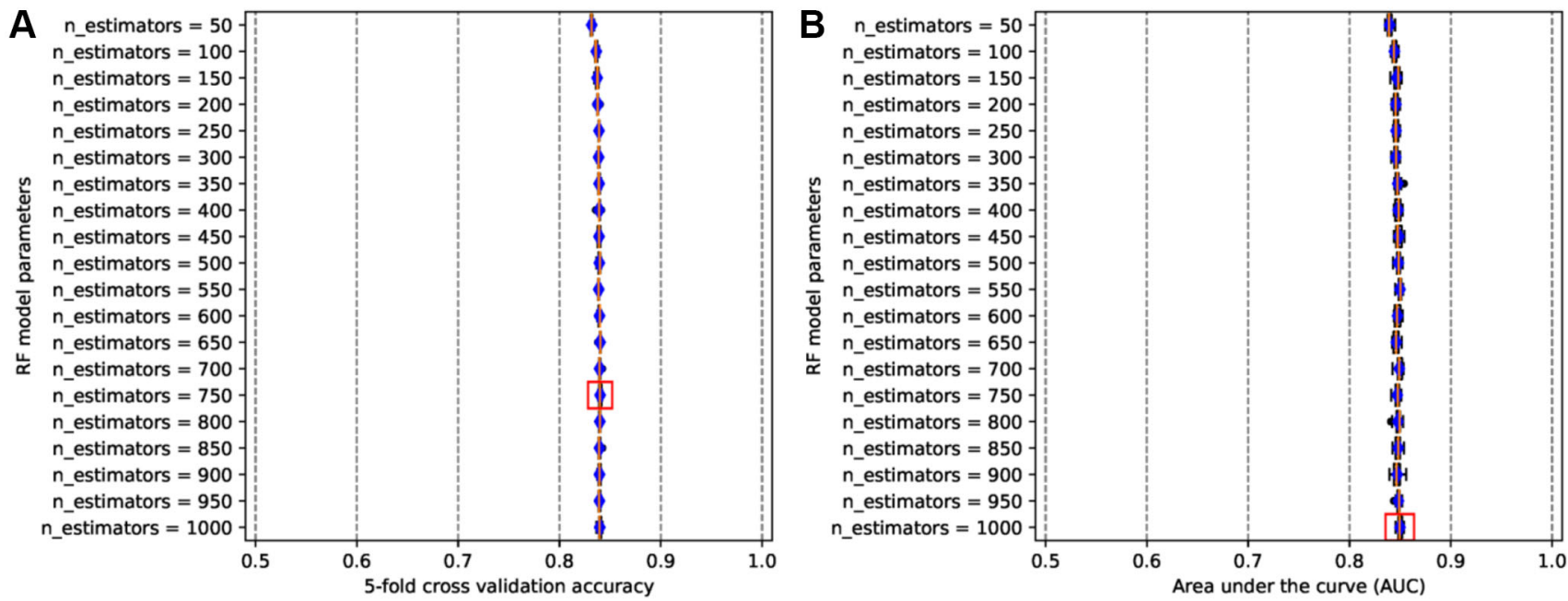

Supplementary Figure 2. Parameter optimization of the random forest (RF) model. The optimal parameter "n_estimators" was determined according to the maximum 5-fold cross-validation accuracy (A) and the maximum area under the curve (AUC) (B). The red box indicates the maximum 5-fold cross-validation accuracy or AUC. 

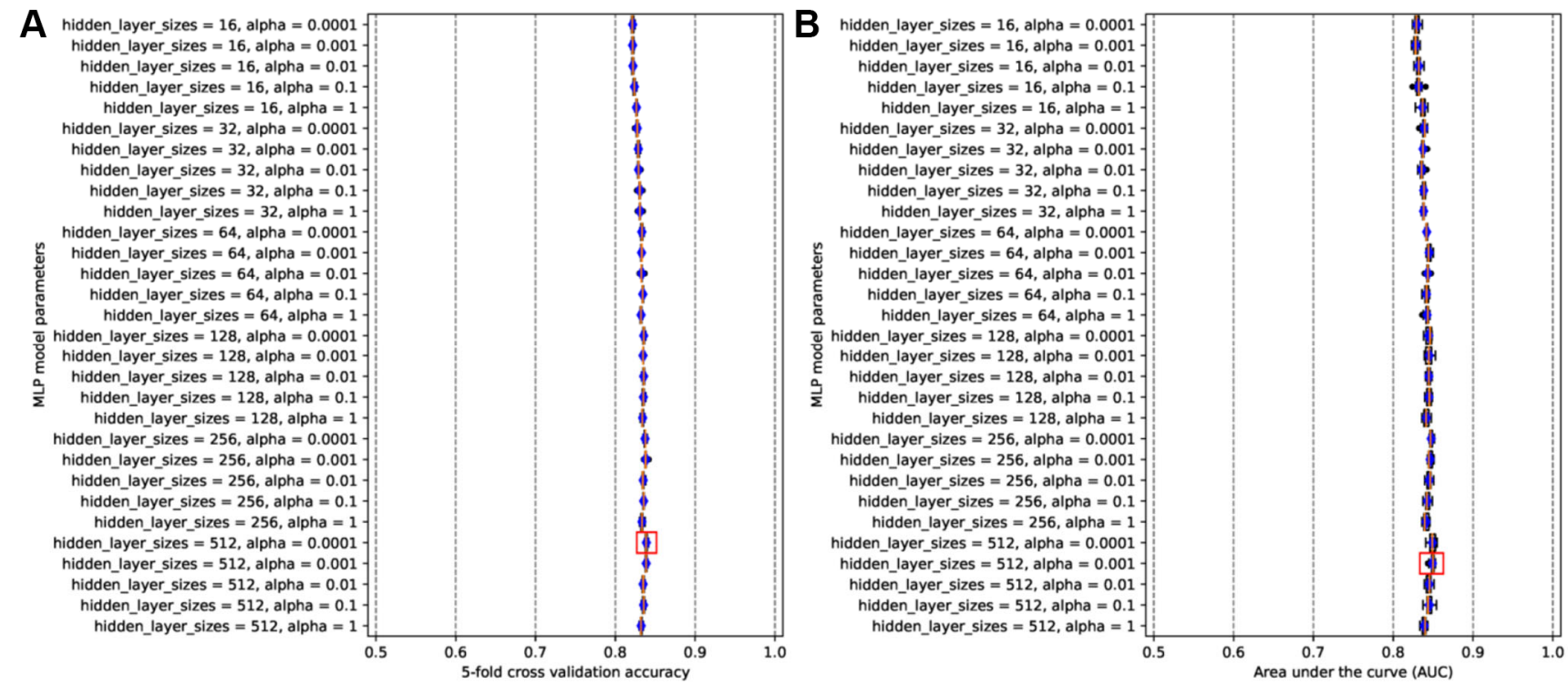

Supplementary Figure 3. Parameter optimization of the multi-layer perception (MLP) model. The optimal parameters "hidden_layer_sizes" and "alpha" were determined according to the maximum 5-fold cross-validation accuracy (A) and the maximum area under the curve (AUC) (B). The red box indicates the maximum 5-fold cross-validation accuracy or AUC.
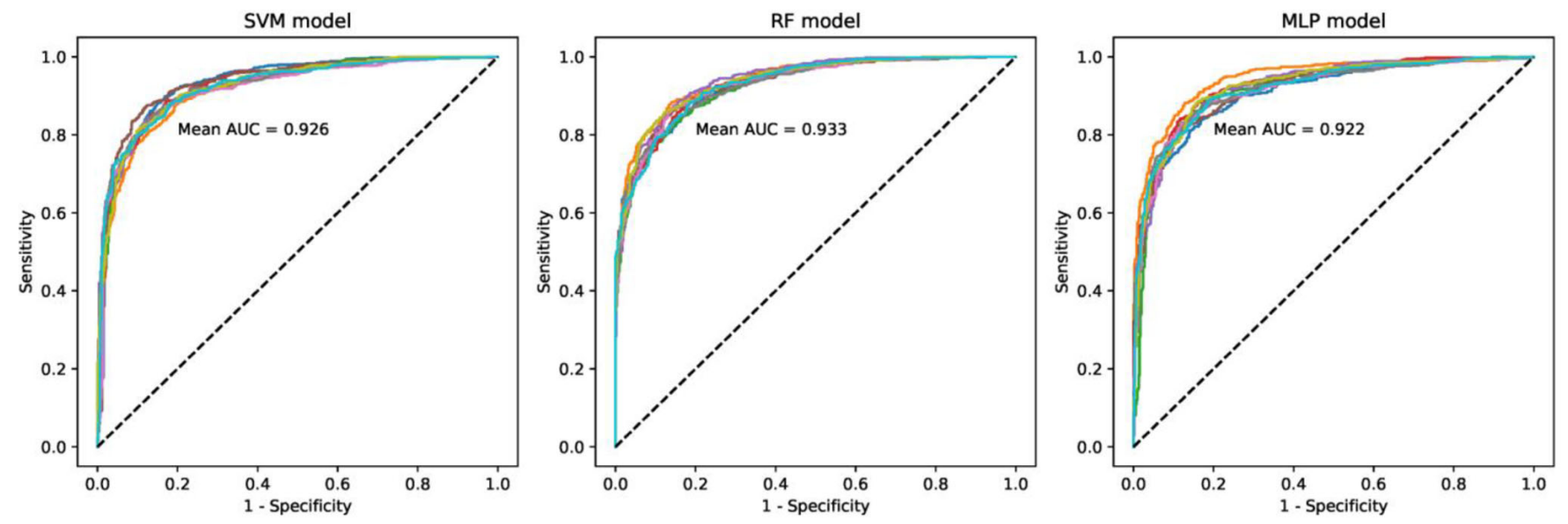

Supplementary Figure 4. The receiver operating characteristic (ROC) curve and area under the curve (AUC) for the optimal SVM, RF, and MLP models. ROC and AUC were calculated for each cross-validation, all 10 times were shown in the figure. The figure showed the use of one of the benchmark datasets (see Methods 2.2 and 2.5) for model construction and its prediction performance. 
A

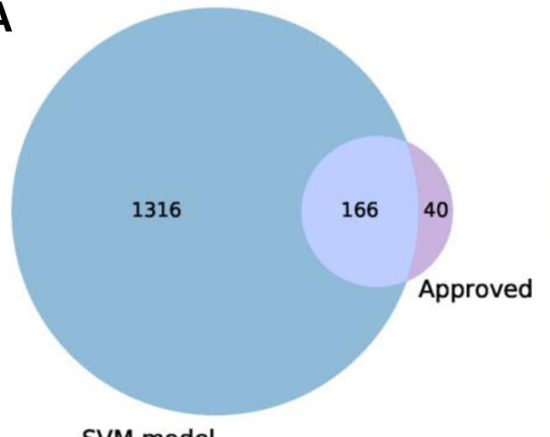

SVM model

B

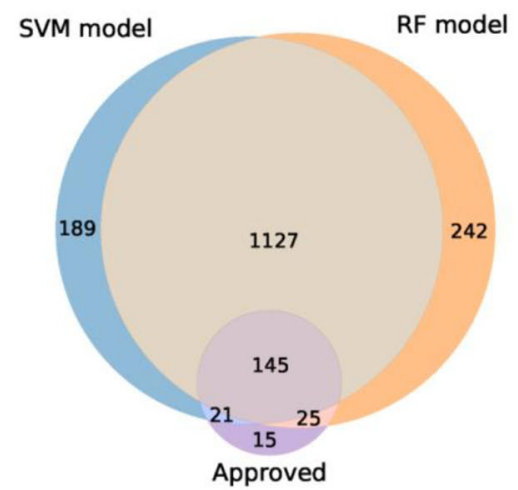

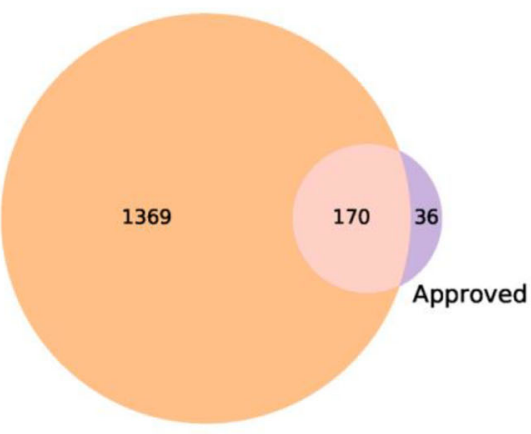

RF model

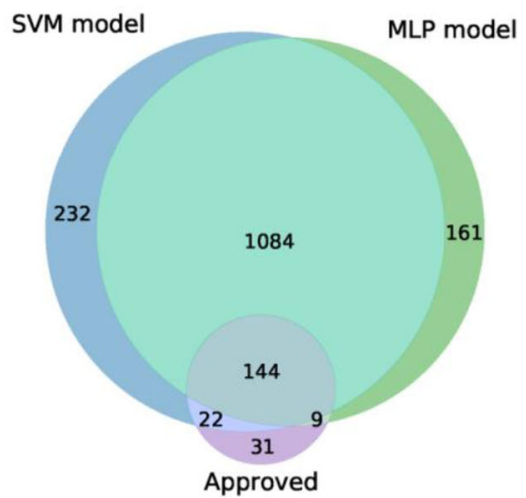

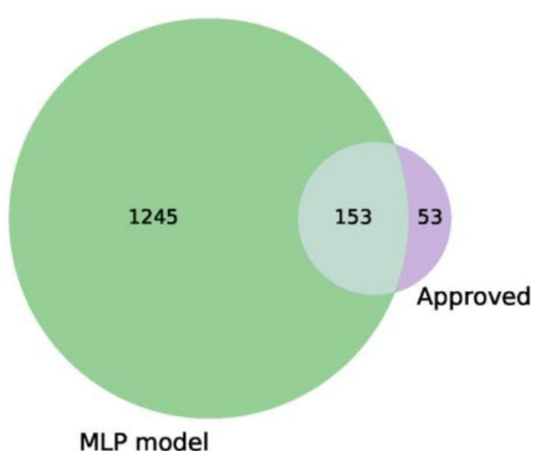

MLP model

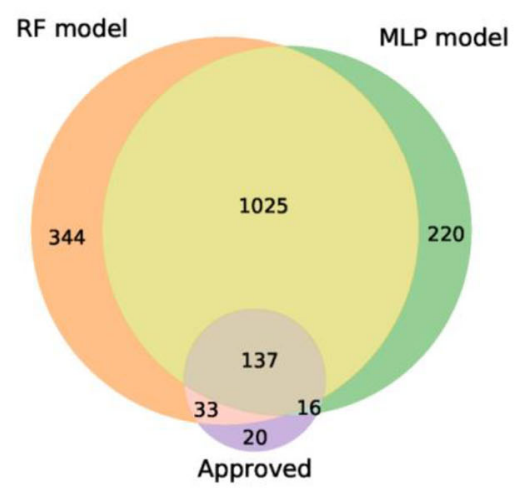

Supplementary Figure 5. Venn diagram of predicted and FDA-approved antibacterial drugs in a single model (A) and the combination of two models (B). 


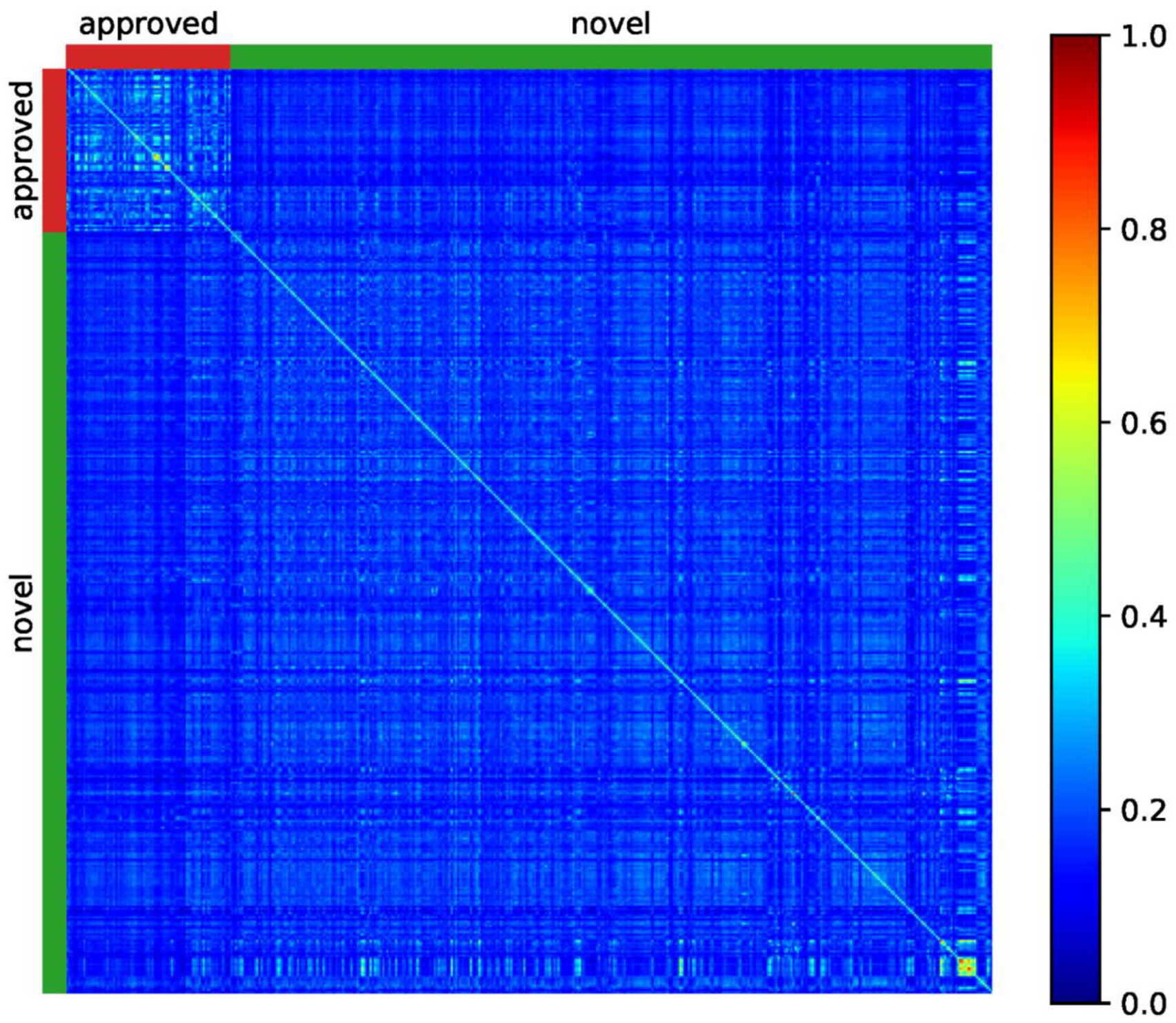

Supplementary Figure 6. FP2 molecular fingerprint similarity between FDA-approved antibacterial drugs and predicted antibacterial compounds. The color bar indicates the similarity from low (0) to high (1). 

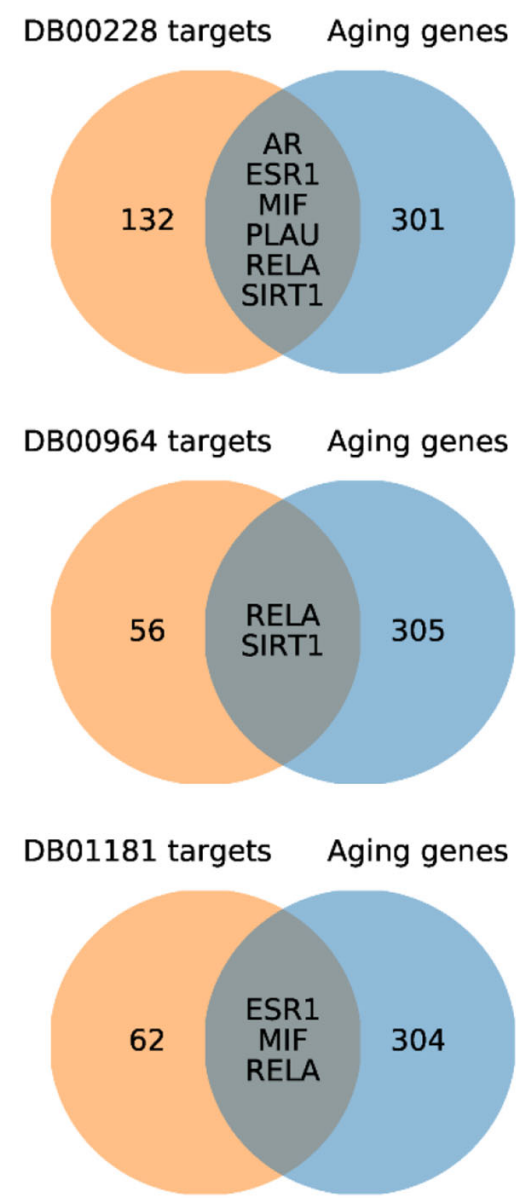
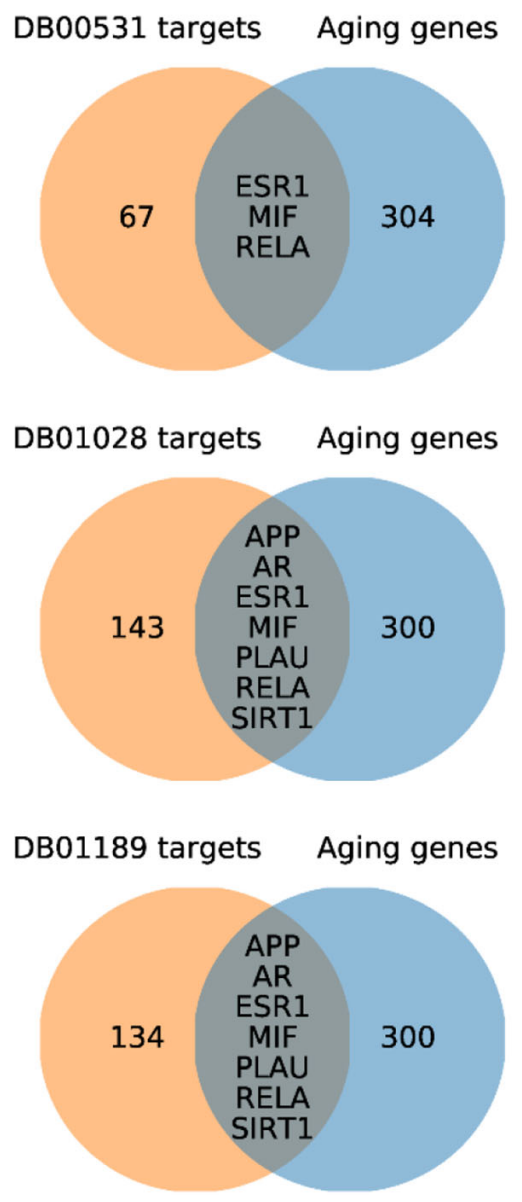

DB00753 targets Aging genes
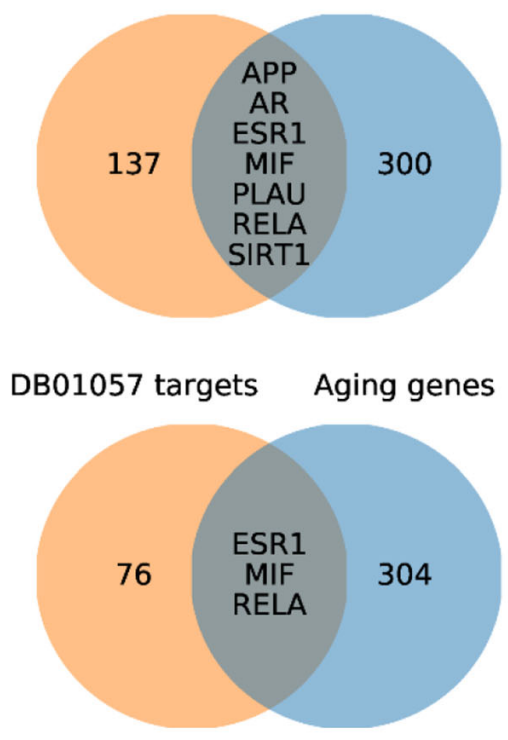

DB01236 targets Aging genes

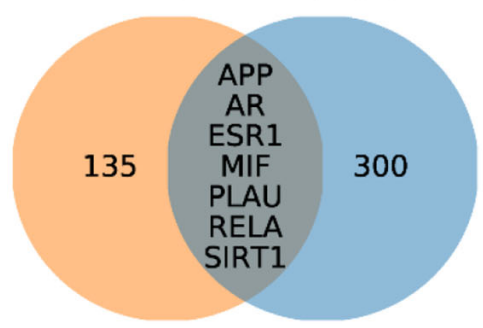

Supplementary Figure 7. Venn diagram of predicted drug targets and human aging-related genes. The union set of SEA, HitPick, and TargetNet predictions were chosen as target genes for the query drug. Human aging-related genes were downloaded from the Human Ageing Genomic Resources (HAGR). 


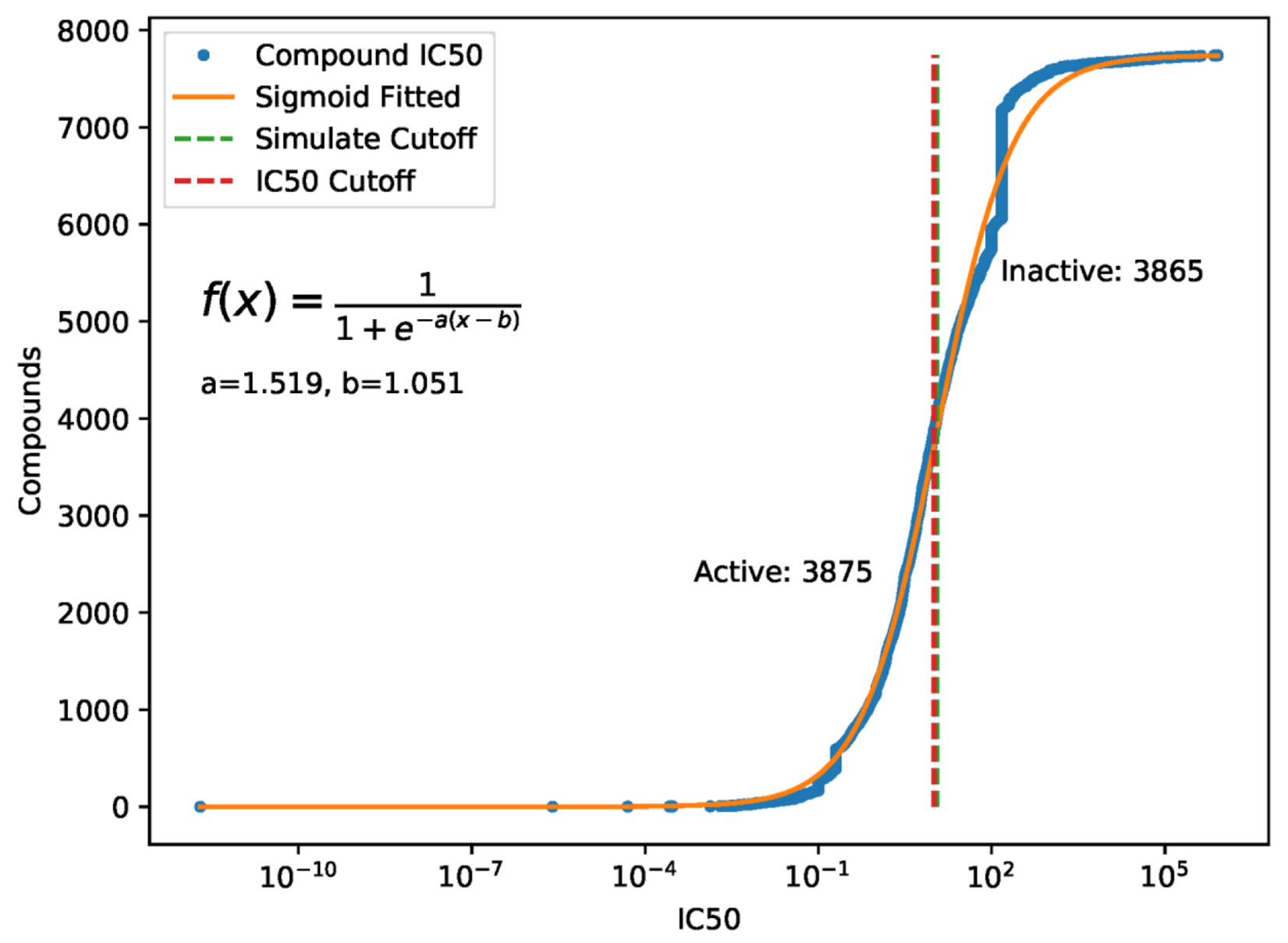

Supplementary Figure 8. Curve fitting of IC50 values of antibacterial compounds. The blue point indicates the IC50 values of all compounds. A sigmoid function was used to perform curve fitting and calculate the fitting parameters (yellow curve). The position of the maximum intercept of the function is defined as the fitting threshold (green dotted line), which is very close to the IC50 cutoff (10 $\mu \mathrm{mol} / \mathrm{L}$ ). Therefore, compounds with IC50 less than $10 \mu \mathrm{mol} / \mathrm{L}$ were defined as active antibacterial compounds, and those with IC50 higher than 10 $\mu \mathrm{mol} / \mathrm{L}$ were considered inactive antibacterial compounds. 


\section{Supplementary Tables}

Supplementary Table 1. The prediction summary of different machine learning models.

\begin{tabular}{lccc}
\hline Model & Number of predicted drugs & Number of overlapped drugs & $\boldsymbol{P}$-value \\
\hline SVM & 1482 & 166 & $1.88 \mathrm{E}-8$ \\
RF & 1539 & 170 & $2.45 \mathrm{E}-8$ \\
MLP & 1398 & 153 & $4.21 \mathrm{E}-6$ \\
SVM, RF & 1272 & 145 & $7.09 \mathrm{E}-7$ \\
SVM, MLP & 1228 & 144 & $8.35 \mathrm{E}-8$ \\
RF, MLP & 1162 & 137 & $2.56 \mathrm{E}-7$ \\
SVM, RF, MLP & 1090 & 133 & $4.24 \mathrm{E}-8$ \\
\hline
\end{tabular}

Abbreviations: SVM: support vector machine; RF: random forest; MLP: multi-layer perception. $P$-values were calculated by the hypergeometric distribution model.

Supplementary Table 2. Core scaffolds and representative drugs of antibacterial compounds.

\begin{tabular}{|c|c|c|c|c|}
\hline Categories & Core Structure & Represented & Name & Structure \\
\hline Quinolones & & DB00218 & Moxifloxacin & \\
\hline Penicillins & & DB00578 & Carbenicillin & \\
\hline Oxazolidinones & & DB00601 & Linezolid & \\
\hline$\beta$-lactams & & DB00689 & Cephaloglycin & \\
\hline Sulfonamides & & DB01124 & Tolbutamide & \\
\hline Lincosamides & & DB01190 & Clindamycin & \\
\hline
\end{tabular}


Carbapenems
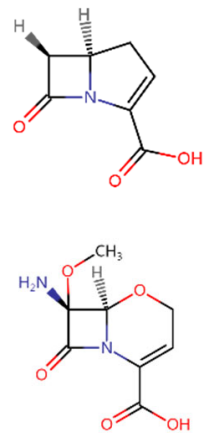

DB01598

Imipenem

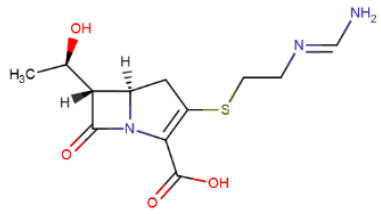

DB04570

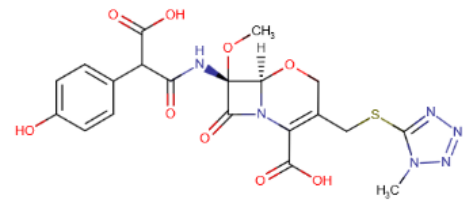

Supplementary Table 3. Novel predicted antibacterial drugs with high similarity to core scaffolds.

\begin{tabular}{|c|c|c|c|c|c|c|}
\hline Categories & Predicted Drug & Query Size & $\begin{array}{c}\text { Target } \\
\text { Size }\end{array}$ & MCS Size & $\begin{array}{c}\text { Tanimoto } \\
\text { Coefficient }\end{array}$ & $\begin{array}{c}\text { Overlap } \\
\text { Coefficient }\end{array}$ \\
\hline Quinolones & DB08820 & 11 & 29 & 11 & 0.38 & 1.00 \\
\hline Sulfonamides & DB00222 & 14 & 34 & 14 & 0.41 & 1.00 \\
\hline Sulfonamides & DB01016 & 14 & 33 & 14 & 0.42 & 1.00 \\
\hline Sulfonamides & DB01067 & 14 & 31 & 14 & 0.45 & 1.00 \\
\hline Sulfonamides & DB01251 & 14 & 37 & 14 & 0.38 & 1.00 \\
\hline Oxazolidinones & DB00315 & 6 & 21 & 6 & 0.29 & 1.00 \\
\hline Oxazolidinones & DB00660 & 6 & 16 & 6 & 0.38 & 1.00 \\
\hline Oxazolidinones & DB06228 & 6 & 29 & 6 & 0.21 & 1.00 \\
\hline Sulfonamides & DB00559 & 14 & 39 & 13 & 0.33 & 0.93 \\
\hline Sulfonamides & DB08439 & 14 & 26 & 13 & 0.48 & 0.93 \\
\hline Quinolones & DB00385 & 11 & 51 & 10 & 0.19 & 0.91 \\
\hline Quinolones & DB00445 & 11 & 39 & 10 & 0.25 & 0.91 \\
\hline Quinolones & DB00524 & 11 & 24 & 10 & 0.40 & 0.91 \\
\hline Quinolones & DB00670 & 11 & 26 & 10 & 0.37 & 0.91 \\
\hline Quinolones & DB00694 & 11 & 38 & 10 & 0.26 & 0.91 \\
\hline Quinolones & DB00695 & 11 & 21 & 10 & 0.45 & 0.91 \\
\hline Quinolones & DB00796 & 11 & 45 & 10 & 0.22 & 0.91 \\
\hline Quinolones & DB00904 & 11 & 22 & 10 & 0.43 & 0.91 \\
\hline Quinolones & DB00963 & 11 & 20 & 10 & 0.48 & 0.91 \\
\hline Quinolones & DB00997 & 11 & 39 & 10 & 0.25 & 0.91 \\
\hline Quinolones & DB01009 & 11 & 19 & 10 & 0.50 & 0.91 \\
\hline Quinolones & DB01022 & 11 & 33 & 10 & 0.29 & 0.91 \\
\hline Quinolones & DB01117 & 11 & 26 & 10 & 0.37 & 0.91 \\
\hline Quinolones & DB01148 & 11 & 29 & 10 & 0.33 & 0.91 \\
\hline Quinolones & DB01177 & 11 & 36 & 10 & 0.27 & 0.91 \\
\hline Quinolones & DB01204 & 11 & 32 & 10 & 0.30 & 0.91 \\
\hline Quinolones & DB01205 & 11 & 22 & 10 & 0.43 & 0.91 \\
\hline Quinolones & DB01325 & 11 & 18 & 10 & 0.53 & 0.91 \\
\hline Quinolones & DB01419 & 11 & 42 & 10 & 0.23 & 0.91 \\
\hline
\end{tabular}




\begin{tabular}{lllllll} 
Quinolones & DB01698 & 11 & 43 & 10 & 0.23 & 0.91 \\
Quinolones & DB0266 & 11 & 20 & 10 & 0.48 & 0.91 \\
Quinolones & DB04880 & 11 & 17 & 10 & 0.56 & 0.91 \\
Quinolones & DB05239 & 11 & 30 & 10 & 0.32 & 0.91 \\
Quinolones & DB06193 & 11 & 24 & 10 & 0.40 & 0.91 \\
Quinolones & DB06207 & 11 & 35 & 10 & 0.28 & 0.91 \\
Quinolones & DB08822 & 11 & 42 & 10 & 0.23 & 0.91 \\
Quinolones & DB08881 & 11 & 33 & 10 & 0.29 & 0.91 \\
Quinolones & DB08911 & 11 & 37 & 10 & 0.26 & 0.91 \\
Quinolones & DB08995 & 11 & 43 & 10 & 0.23 & 0.91 \\
Quinolones & DB09079 & 11 & 40 & 10 & 0.24 & 0.91 \\
Quinolones & DB09183 & 11 & 35 & 10 & 0.28 & 0.91 \\
Quinolones & DB09214 & 11 & 19 & 10 & 0.50 & 0.91 \\
Quinolones & DB11363 & 11 & 36 & 10 & 0.27 & 0.91 \\
Quinolones & DB11577 & 11 & 28 & 10 & 0.34 & 0.91 \\
Quinolones & DB11689 & 11 & 27 & 10 & 0.36 & 0.91 \\
Quinolones & DB11699 & 11 & 21 & 10 & 0.45 & 0.91 \\
Quinolones & DB11967 & 11 & 27 & 10 & 0.36 & 0.91 \\
Quinolones & DB11986 & 11 & 41 & 10 & 0.24 & 0.91 \\
Quinolones & DB13225 & 11 & 22 & 10 & 0.43 & 0.91 \\
Quinolones & DB15477 & 11 & 30 & 10 & 0.32 & 0.91 \\
Lincosamides & DB09419 & 24 & 10 & 9 & 0.36 & 0.90 \\
\hline
\end{tabular}

Abbreviation: MCS: maximum common substructure. Tanimoto Coefficient = MCS Size/(Query Size + Target Size - MCS Size) Overlap Coefficient $=$ MCS Size/min(Query Size, Target Size). The MCS algorithm was used to calculate structural similarities among small molecules. A total of 957 predicted novel antibacterial drugs were calculated among 8 core structures. The table showed 51 predicted drugs with an overlap coefficient $>0.9$ among 8 core structures, the results are sorted by overlap coefficient from high to low.

Supplementary Table 4. Details of the 9 predicted novel antibacterial drugs.

Drug ID


DB00753

Isoflurane

Apraclonidine

DB00964

Apraclonidine

DB01028

Methoxyflurane<smiles>Nc1cc(Cl)c(NC2=NCCN2)c(Cl)c1</smiles>
substituted derivatives

DB01057

Echothiophate

DB01181

Ifosfamide
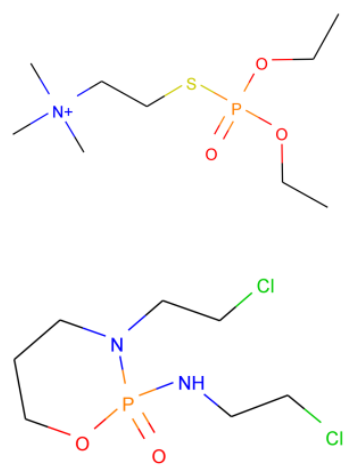

Oxazaphosphinanes

Organofluorides
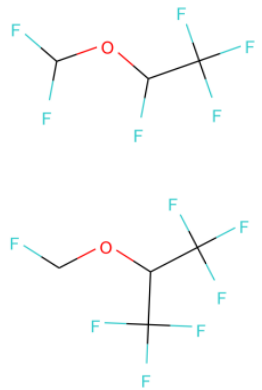

Benzene and

Organonitrogen compounds

General anesthesia

Ocular hypertension

Postsurgical ocular hypertension

Organooxygen
compounds

General anesthesia

Accommodative component in esotropia

Chronic angle-closure glaucoma

Open-angle glaucoma Nonuveitic secondary glaucoma

Germ cell testicular cancer

Cervical cancer

Soft tissue sarcomas

Osteosarcoma

Bladder cancer

Ovarian cancer

Small cell lung cancer

Non-Hodgkin's lymphoma

General anesthesia Maintenance of anesthesia therapy

Organooxygen compounds

General anesthesia

Supplementary Table 5. Binary bits of different types of molecular fingerprints or vector features were used for machine learning modeling.

\begin{tabular}{lcc}
\hline Compound Features & Description & Number of features \\
\hline Molecular fingerprints & & \\
FP2 & FP2 Fingerprints & 1024 \\
FP3 & FP3 Fingerprints & 210 \\
FP4 & FP4 Fingerprints & 307 \\
DLFP & Daylight-like Fingerprints & 2048 \\
MACCS & MACCS keys & 166 \\
ECFP2 & Extended-Connectivity Fingerprints, Iteration 1 \\
ECFP4 & Extended-Connectivity Fingerprints, Iteration 2 & 1024 \\
& & 1024 \\
\hline
\end{tabular}


ECFP6

FCFP2

FCFP4

FCFP6

Vector features

mol2vec

SMILES2Vec

FP2VEC
Extended-Connectivity Fingerprints, Iteration 3

1024

Functional-Class Fingerprints, Iteration 1

1024

Functional-Class Fingerprints, Iteration 2

1024

Functional-Class Fingerprints, Iteration 3

1024

Vector features based on Morgan fingerprints

200

Vector features based on molecule SMILES

100

Trainable embedding vectors based on fingerprints

100 